\title{
Synthesis of novel 1,2,3-triazole-based hybrids via click reactions
}

\author{
Samvel N. Sirakanyan, ${ }^{\text {a }}$ Tariel V. Ghochikyan, ${ }^{\mathrm{b}}$ Domenico Spinelli, ${ }^{* c}$ Armen S. Galstyan, ${ }^{\mathrm{b}}$ Athina \\ Geronikaki, ${ }^{* d}$ Melanya A. Samvelyan, ${ }^{b}$ Elmira K. Hakobyan, ${ }^{a}$ Anush A. Hovakimyan ${ }^{a}$
}

${ }^{a}$ Scientific Technological Center of Organic and Pharmaceutical Chemistry of National Academy of Science of Republic of Armenia, Institute of Fine Organic Chemistry of A.L.Mnjoyan, Armenia 0014, Yerevan, Ave.

Azatutyan 26

${ }^{b}$ Faculty of Chemistry, Yerevan State University, Alex Manoogian 1, 0025 Yerevan, Armenia ${ }^{c}$ Dipartimento di Chimica G. Ciamician, Alma Mater Studiorum-Università di Bologna, Via F. Selmi 2, Bologna 40126, Italy

${ }^{d}$ Aristotle University of Thessaloniki, School of Pharmacy, Thessaloniki 54124, Greece Email:geronik@pharm.auth.gr;shnnr@mail.ru

\section{Dedicated to Professor Girolamo Cirrincione}

Received 04-21-2021

Accepted Manuscript 09-21-2021

Published on line 10-09-2021

\section{Abstract}

1,2,3-Triazoles have attracted the interest of researchers due to their wide range of biological activities which include antitumor, anti-leishmanial, bioluminescent and fungicidal activities This paper describes the synthesis of novel triazole hybrids containing biologically active fragments through cycloaddition (click) reactions, with the aim of increasing the diversity of known and active 1,2,3-triazole derivatives. All new compounds have been characterized by physicochemical methods.

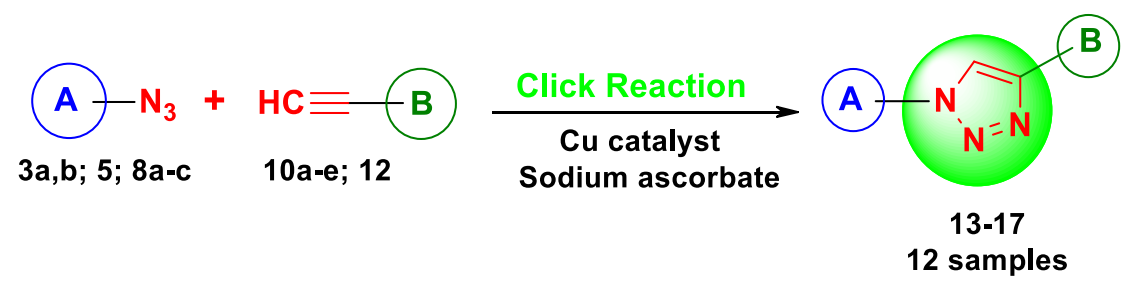

Keywords: Condensed pyridines, spirodibutanolides, thiopropargyl-1,2,4-triazoles, 1,2,3-triazoles, click reaction 


\section{Introduction}

Since many drugs used in medical therapy contain heterocycles in their structure, the introduction in their structure of triazole rings, especially 1,2,3-triazoles, has attracted special attention. The syntheses and the behavior of these heterocyclic compounds have been intensely investigated in the last decades - research into the development of new methods for the synthesis of substituted triazoles and identification of their possible biological/pharmacological properties thus proceed with great interest. In fact, several new methods for producing various 1,2,3-triazole derivatives ${ }^{1,2}$ have been recently proposed, and it has been shown that the novel compounds derived through this approach have a wide spectrum of biological properties/activities. Among these bioactivities, cytotoxic, ${ }^{3}$ antitumor, ${ }^{4}$ leishmanicidal, ${ }^{5}$ bioluminescent ${ }^{6}$ and fungicidal ${ }^{7}$ activities should be noted, all which have been shown to be characteristic of certain 1,2,3-triazoles. The analysis of literature data shows that the nature of substituents on the triazole cycle is very important. Thus, modification of these substituents is expected to result in significant changes in the biological properties of 1,2,3-triazole 44derivatives thus formed. For this reason, research into the development of methods for the synthesis of novel substituted triazoles is constantly increasing. ${ }^{8,9}$ Of particular value are studies on the isolation and investigation of substances from natural raw materials that possess cytotoxic, ${ }^{10}$ antitumor ${ }^{11}$ activities, or being inhibitors of topoisomerase, ${ }^{12}$ DPP-4 and cancer cell growth. ${ }^{13}$

The main method to prepare 1,2,3-triazoles involves the 1,3-cycloaddition of organic azides with a terminal triple bond, via a click reaction. ${ }^{14-16}$ In this paper we propose the synthesis of novel triazole-linked hybrids containing biologically active fragments, with the aim to increase the structural range of known and active 1,2,3-triazole derivatives.

It is known that disubstituted piperazines can exhibit a wide range of pharmacological activity; they can act as anticonvulsant, ${ }^{17}$ antifungal, ${ }^{18}$ antiviral, ${ }^{19}$ radioprotective, ${ }^{20}$ antibacterial and antimalarial ${ }^{21,22}$ agents. Accordingly, our studies in this field have shown that piperazine derivatives of pyrano[3,4-c]pyridines display high antibacterial ${ }^{23}$ and neurotropic activity. ${ }^{24,25}$ In addition, bicyclic pyrano[3,4-c]pyridines synthesized by us were characterized by eliciting pronounced cardiotonic activities. ${ }^{26}$ Thus, it was our intent to incorporate these moieties (types of structures) into the click-derived compounds, which also included examples containing dioxaspiro[4.4]nonane-1,6-dionesand carvone-derived terpenes.

\section{Results and Discussion}

As starting compounds for the synthesis of the first set of required azides, two ethyl 3-allyl-5-methyl-2oxotetrahydrofuran-3-carboxylates $\mathbf{1}^{27}$ were used. The reaction of compounds 1 with bromine led to the cyclization of the second tetrahydrofuran ring resulting in the formation of 3-(bromomethyl)-8-methyl-2,7dioxaspiro[4.4]nonane-1,6-diones $\mathbf{2 a , b}$ as we have previously reported. ${ }^{27}$ These results are in good agreement with the Baldwin rules for ring formation. In the case of nucleophilic cyclizations, both 5-exo-tet and 6-exo-tet cyclizations are favourable. ${ }^{28,29}$ However, it was shown by Houk that $\gamma$-butyrolactone is less strained than $\delta$ valerolactone; therefore, this cyclization is a proposed to be a thermodynamically controlled process. ${ }^{30,31}$ Finally, the compounds $\mathbf{2}$ were converted into the desired 3-(azidomethyl)-3,8,8-trimsubstituted-2,7dioxaspiro[4.4]nonane-1,6-diones $\mathbf{3 a , b}$ (Scheme 1 ) by treatment with sodium azide in DMSO at room temperature. Compounds $\mathbf{2}$ and $\mathbf{3}$ were found to be inseparable mixtures of diastereomers in 1:1-1.7:1 ratio, as shown by their NMR spectra. 


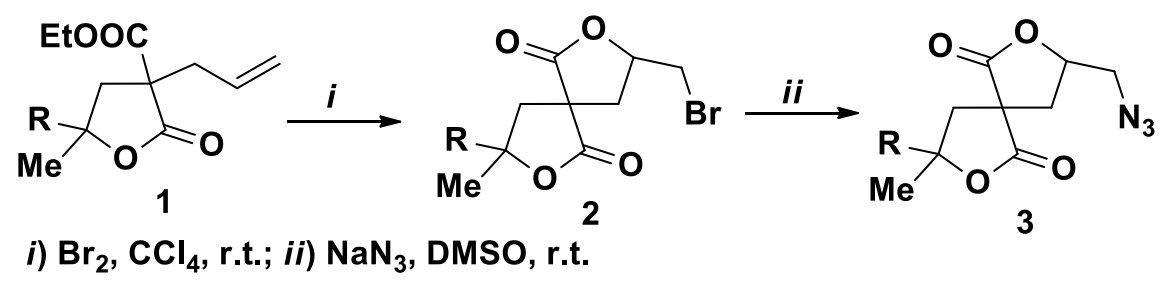

1-3. $\mathbf{a}: \mathrm{R}=\mathrm{H}$; b: $\mathrm{R}=\mathrm{Me}$.

Scheme 1. Synthesis of 3-(azidomethyl)-3,8,8-trimsubstituted-2,7-dioxaspiro[4.4]nonane-1,6-diones 3. Yields, \%: 83 (2a); 86 (2b); 80 (3a); 83 (3b).

Next, the synthesis of the azide 5 derived from the L-carvone, (R)-5-(3-azidochloroprop-1-en-2-yl)-2methylcyclohex-2-enone (4) was performed as described in Scheme 2. According to our previous work in collaboration, 1,2,3-triazoles obtained using triazole 5 displayed pronounced antioxidant and fluorescent properties. $^{32}$

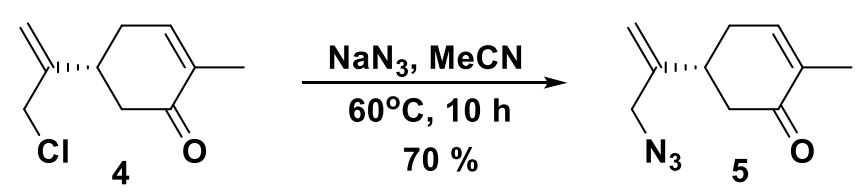

Scheme 2. Synthesis of (R)-5-(3-azido-prop-1-en-2-yl)-2-methylcyclohex-2-enone 5.

Finally, to add a piperazine group to the structure of the envisaged 1,2,3-triazoles we also synthesized some azide derivatives 8 according to Scheme 3. Thus, by the acylation of 3(6)-piperazine derivatives of fused pyridines $6^{23,24}$ with chloroacetyl chloride, the relevant 1(8)-alkyl-3(6)-[4-(chloroacetyl)piperazin-1-yl]pyridine4(5)-carbonitriles 7 were obtained. Reaction of these compounds with sodium azide led to the formation of 1(8)-alkyl-3(6)-[4-(azidoacetyl)piperazin-1-yl][c]pyridine-4(5)-carbonitriles 8 in high yields (Table 1).

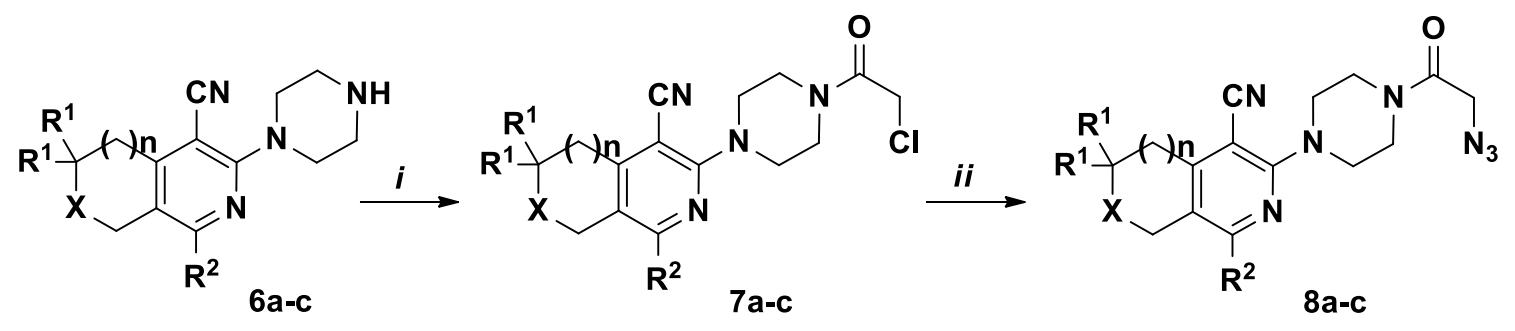

i) $\mathrm{ClCH}_{2} \mathrm{COCl}, \mathrm{C}_{6} \mathrm{H}_{6}, \mathrm{Et}_{3} \mathrm{~N}, 35^{\circ} \mathrm{C}, 6 \mathrm{~h}$; ii) $\mathrm{NaN}_{3}$, MeCOMe, reflux $15 \mathrm{~h}$

Scheme 3. Synthesis of 3(6)-[4-(azidoacetyl)piperazin-1-yl][c]pyridine-4(5)-carbonitriles 8. 
Table 1. 3(6)-[4-(Azidoacetyl)piperazin-1-yl]pyridines 8

\begin{tabular}{llllll}
\hline Compound & $\mathrm{X}$ & $\mathrm{R}^{1}$ & $\mathrm{n}$ & $\mathrm{R}^{2}$ & Yield (\%) \\
\hline $\mathbf{8 a}$ & $\mathrm{CH}_{2}$ & $\mathrm{H}$ & 0 & $i-\mathrm{Bu}$ & 74 \\
$\mathbf{8 b}$ & $\mathrm{O}$ & $\mathrm{Me}$ & 1 & $\mathrm{Et}$ & 81 \\
$\mathbf{8 c}$ & $\mathrm{O}$ & $\mathrm{Me}$ & 1 & $\mathrm{Pr}$ & 83 \\
\hline
\end{tabular}

The next step for the preparation of the title hybrids required the introduction of terminal alkynes to suitable scaffolds. Thus, by reaction of the 3(6)-hydroxy derivatives of cyclopenta[c]pyridine 9a, 5,6,7,8tetrahydroisoquinolines $\mathbf{9 b}, \mathrm{c}^{33}$ and of pyrano[3,4-c]pyridines $\mathbf{9 d}, \mathrm{e}^{34}$ with propargyl bromide, the relevant $O$ alkylated derivatives $\mathbf{1 0 a - e}$ were synthesized in reasonable yields (Scheme 4, Table 2).
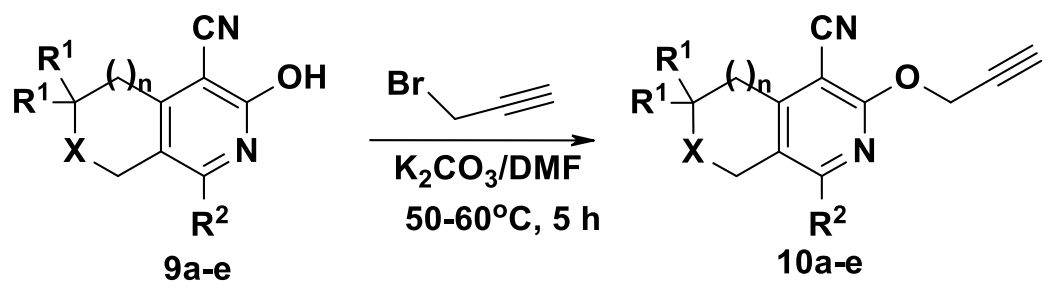

Scheme 4. Synthesis of 1(8)-alkyl-3(6)-(prop-2-yn-1-yloxy)pyridine-4(5)-carbonitriles 10.

Table 2. 1(8)-Alkyl-3(6)-(prop-2-yn-1-yloxy)pyridines 10

\begin{tabular}{cccccc}
\hline Compound & $\mathrm{X}$ & $\mathrm{n}$ & $\mathrm{R}^{1}$ & $\mathrm{R}^{2}$ & Yield (\%) \\
\hline 10a & $\mathrm{CH}_{2}$ & 0 & $\mathrm{H}$ & $i-\mathrm{Pr}$ & 76 \\
10b & $\mathrm{CH}_{2}$ & 1 & $\mathrm{H}$ & $i-\mathrm{Pr}$ & 84 \\
10c & $\mathrm{CH}_{2}$ & 1 & $\mathrm{H}$ & $\mathrm{Ph}$ & 62 \\
10d & $\mathrm{O}$ & 1 & $\mathrm{Me}$ & $\mathrm{Me}$ & 65 \\
10e & $\mathrm{O}$ & 1 & $\mathrm{Me}$ & $i-\mathrm{Pr}$ & 87 \\
\hline
\end{tabular}

To increase the structural range of the obtained compounds containing a triple bond, we also synthesized thiopropargyl-1,2,4-triazoles 12 from the corresponding 5-aryl-4-phenyl-4H-1,2,4-triazole-3-thiols 1135 (Scheme 5), which, as mentioned above, have been shown to be physiologically active substances.

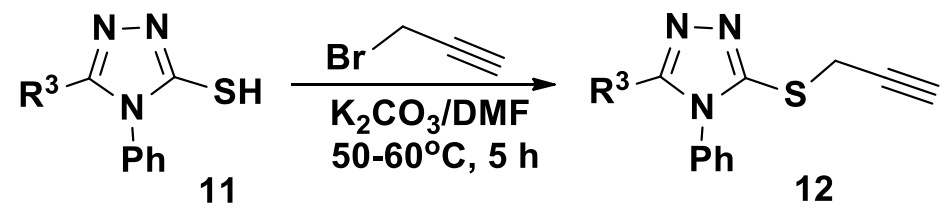

11,12. a: $\mathrm{R}^{3}=2$-furyl; b: $\mathrm{R}^{3}=0-\mathrm{MeOC}_{6} \mathrm{H}_{4}$.

Scheme 5. Synthesis of 4-phenyl-5-(prop-2-yn-1-ylthio)-4H-1,2,4-triazoles 12. Yields, \%: 75 (12a); 80 (12b).

Finally, the desired hybrid compounds were prepared by a Cu-catalyzed azide-alkyne click reaction ${ }^{14-16}$ between the propargylated compounds 10/12 and azido derivatives 3, 5 and $\mathbf{8}$ (Scheme 6). As reductant, ascorbic acid was used in most of the syntheses of the triazole derivatives 13-17, which were obtained in moderate/high yields (Yields $=55-76 \%$; Table 3). 


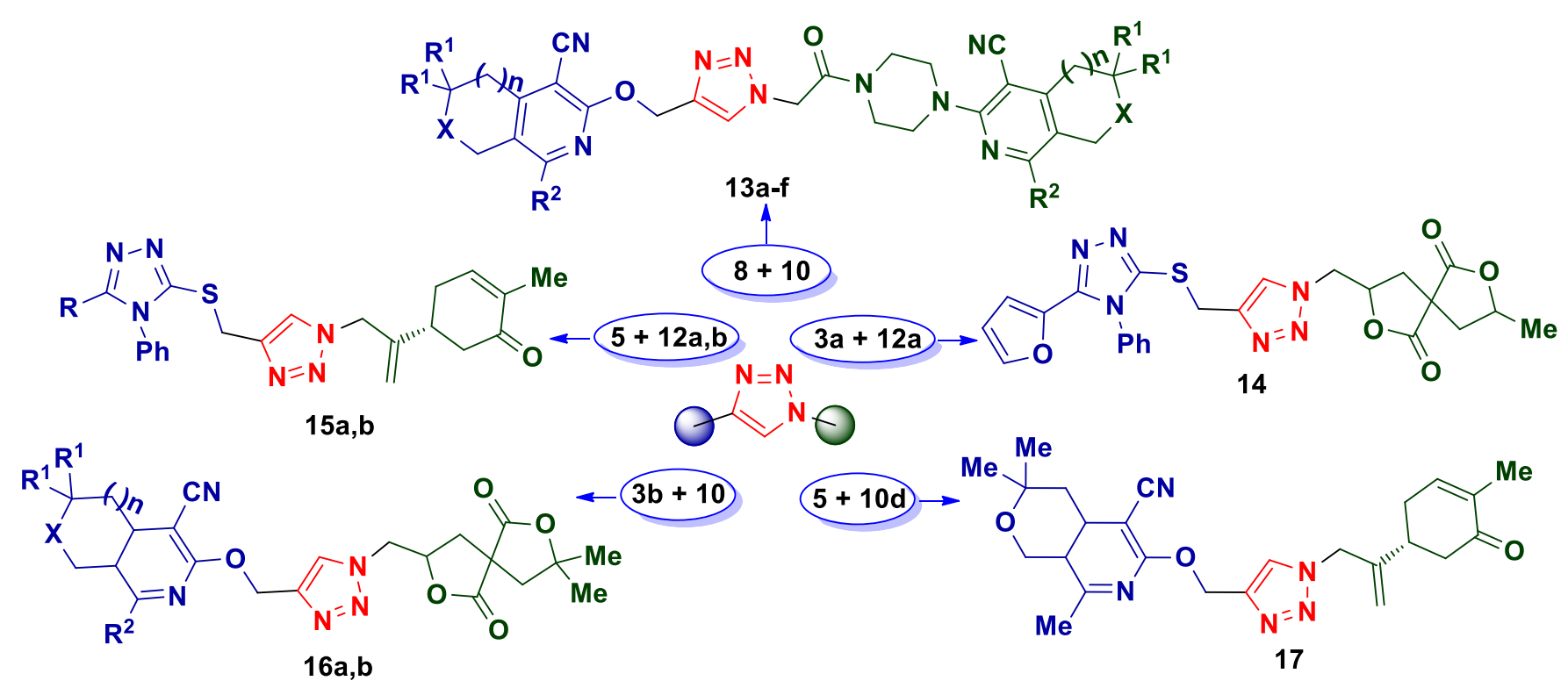

Scheme 6. Synthesis of target hybrids 13-17 via click reaction.

Table 3. Synthesis of aimed hybrids 13-17

Compound


Table 3. Continued

$13 e$

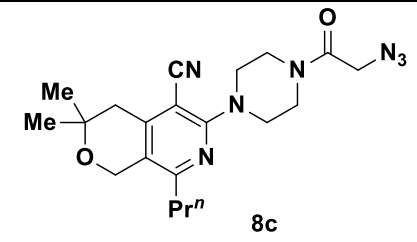

$13 f$

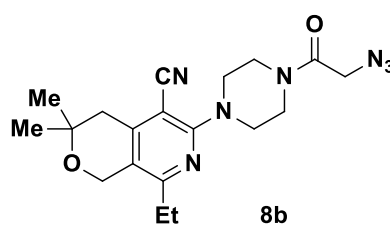

14<smiles>CC1CC2(CC(CN)OC2=O)C(=O)O1</smiles>

$15 a$<smiles>C=C(CN)[C@H]1CC=C(C)C(=O)C1</smiles>

$15 b$<smiles>C=C(CN)[C@H]1CC=C(C)C(=O)C1</smiles>

5<smiles>CC1(C)CC2(CC(CN)OC2=O)C(=O)O1</smiles>

$16 a$

$16 b$

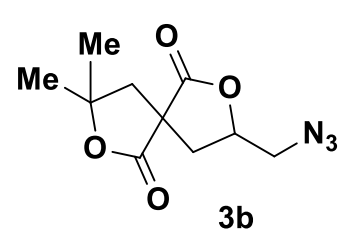

17

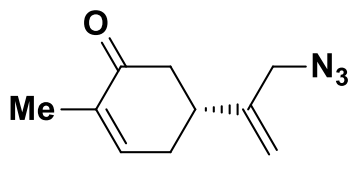

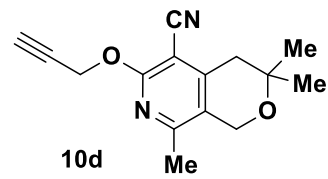

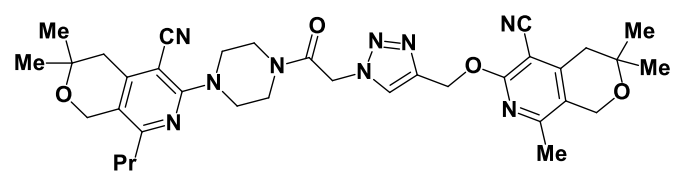

71

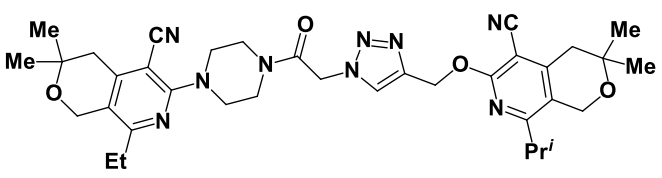

76

$10 \mathrm{e}$
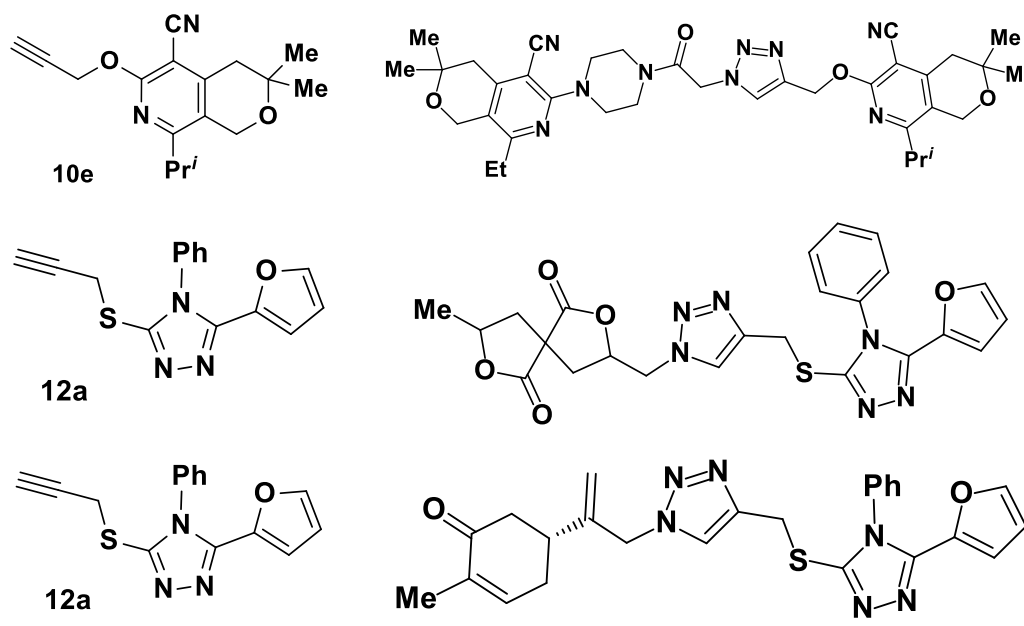

65
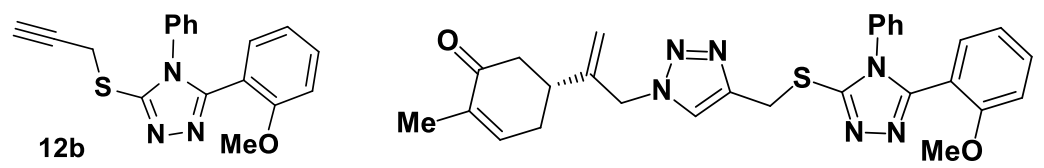

64
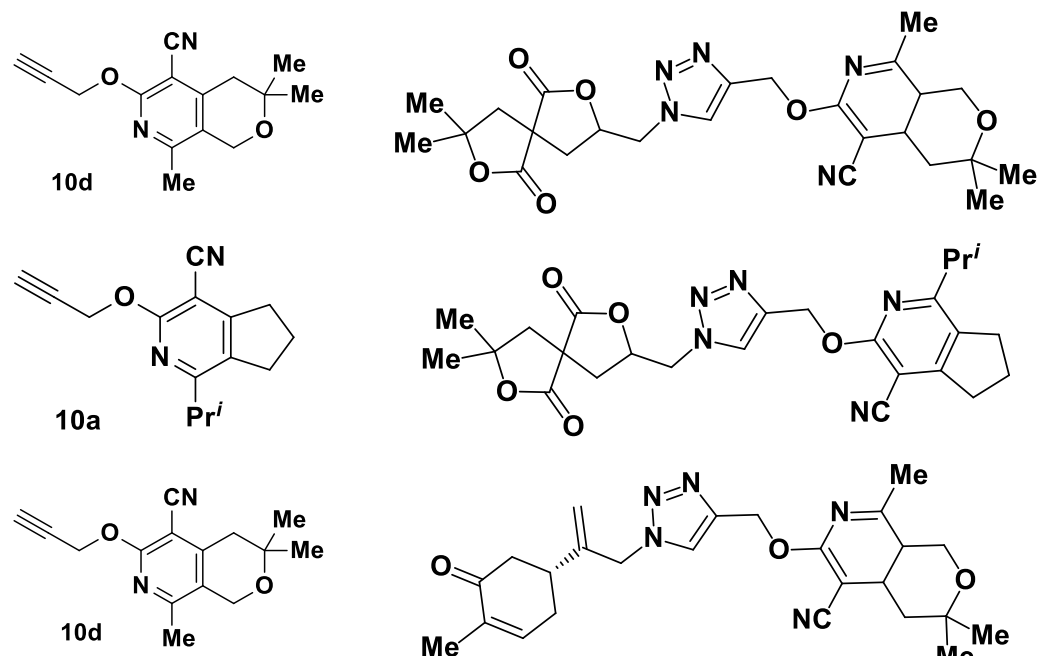

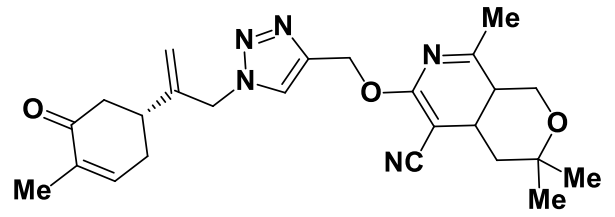

55

The structure of the obtained hybrids 13-17 was confirmed by IR and NMR spectroscopy and by elemental analysis. In the IR spectra of these compounds the absorption bands of the azido group and of triple bond at 2095-2107 $\mathrm{cm}^{-1}$ (typical for the starting 3, 5, 8) and at 2115-2133; 3249-3283 $\mathrm{cm}^{-1}$ (typical for the starting 10 and 12), respectively, were absent. In addition, the ${ }^{1} \mathrm{H}$ NMR spectra of compounds 13-17 did not show the triplet signal of the $\mathrm{CH}$ group at 2.72-2.97 ppm, characteristic for the initial compounds 10 and 12, while the singlet signal attributed to $\mathrm{CH}$ group of the triazole ring was observed at 7.93-7.98 ppm, thus supporting the cyclized structures. As far as starting compounds $\mathbf{3}$ are concerned it should be mentioned that since they are complex structures, containing 2-3 chiral centers, instead of pure products, mixtures of inseparable diastereoisomers were obtained. 


\section{Conclusions}

In summary, we synthesized new 1,2,3-triazole based hybrids with possible interesting biological/pharmacological activities by the Cu-catalyzed click reaction between synthesized alkynes and azides, containing novel chemical frameworks potentially responsible for interesting biological activities. These bioactivities will be ascertained in ongoing studies and will be reported in due course.

\section{Experimental Section}

General. ${ }^{1} \mathrm{H}$ and ${ }^{13} \mathrm{C}$ NMR spectra were recorded in $\mathrm{DMSO} / \mathrm{CCl}_{4}(1 / 3)$ solution $\left(300 \mathrm{MHz}\right.$ for ${ }^{1} \mathrm{H}$ and $75 \mathrm{MHz}$ for ${ }^{13} \mathrm{C}$, respectively) on a Varian Mercury 300VX spectrometer. Chemical shifts were reported as $\delta$ (parts per million) relative to TMS as internal standard. IR spectra were recorded on Nicolet Avatar 330-FT-IR spectrophotometer and the reported wave numbers were given in $\mathrm{cm}^{-1}$. All melting points were determined in an open capillary and were uncorrected. Elemental analyses were performed on a Elemental Analyzer Euro EA 3000. Compounds $2,{ }^{27} \mathbf{5}^{32} \mathbf{6},{ }^{23,24} \mathbf{9},{ }^{33,34} \mathbf{1 1}^{35}$ were already described.

General procedure for the synthesis of azido-lactones (3a,b). To the corresponding bromomethyl-lactone 2 (1 $\mathrm{mmol}$ ) in DMSO $(5 \mathrm{~mL}) \mathrm{NaN}_{3}(1.35 \mathrm{mmol}, 0.088 \mathrm{~g})$ was added and the mixture was stirred for $12 \mathrm{~h}$ at room temperature. After the reaction mixture was poured in water $(60 \mathrm{~mL})$ and extracted with $\mathrm{DCM}(3 \times 15 \mathrm{~mL})$. Combined extracts were dried over $\mathrm{Na}_{2} \mathrm{SO}_{4}$, volatiles were evaporated and the residue was passed through a short silica gel pad in hexane-DCM 1:1 to obtain pure product.

3-(Azidomethyl)-8-methyl-2,7-dioxaspiro[4.4]nonane-1,6-dione (3a). Yield 80\%, viscous oil, $\mathrm{n}_{\mathrm{D}}^{20} 1.4978$. IR

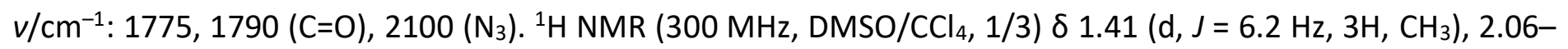
$1.93\left(\mathrm{~m}, 1 \mathrm{H}, \underline{\mathrm{CH}}_{2} \mathrm{CH}\right), 2.20-2.06\left(\mathrm{~m}, 0.5 \mathrm{H}, \mathrm{CH}_{2} \mathrm{CH}\right), 2.63-2.31\left(\mathrm{~m}, 0.5 \mathrm{H}, \underline{\mathrm{CH}}_{2} \mathrm{CH}\right), 2.94-2.66\left(\mathrm{~m}, 2 \mathrm{H}, \mathrm{C} \underline{H}_{2} \mathrm{CH}\right)$, 3.76-3.48 (m, 2H, CH$\left.{ }_{2} \mathrm{~N}\right)$, 4.96-4.65 (m, 2H, CHO). ${ }^{13} \mathrm{C} \mathrm{NMR}\left(75 \mathrm{MHz}, \mathrm{DMSO} / \mathrm{CCl}_{4}, 1 / 3\right) \delta 20.3,20.0,32.9,34.1$. 34.2, 35.7, 39.3, 40.1, 40.4, 52.2, 52.6, 52.9, 53.0, 74.6, 74.7, 75.1, 76.6, 76.9, 172.6, 172.5, 172.8, 172.8. Anal. calcd. for $\mathrm{C}_{9} \mathrm{H}_{11} \mathrm{~N}_{3} \mathrm{O}_{4}$ : C 48.00; $\mathrm{H} 4.92 ; \mathrm{N} 18.66 \%$. Found: C 47.91; $\mathrm{H} 4.99 ; \mathrm{N} 18.75 \%$.

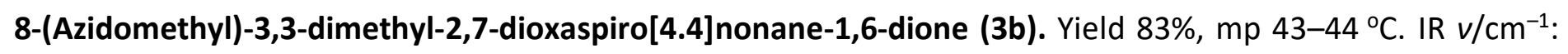
1773, $1787(\mathrm{C}=\mathrm{O}), 2095\left(\mathrm{~N}_{3}\right) .{ }^{1} \mathrm{H} \mathrm{NMR}\left(300 \mathrm{MHz}, \mathrm{DMSO} / \mathrm{CCl}_{4}-1 / 3\right) \delta 1.48\left(\mathrm{~s}, 3 \mathrm{H}, \mathrm{CH}_{3}\right), 1.60-1.50\left(\mathrm{~m}, 3 \mathrm{H}, \mathrm{CH}_{3}\right)$, 2.38-2.17 (m, 1.66H, CH in lactone), 2.72-2.52 (m, 1.68H, CH in lactone), 2.88-2.76 (m, $0.66 \mathrm{H}, \mathrm{CH}_{2}$ in lactone), 3.76-3.51 (m, 2H, CH $2 \mathrm{~N}), 4.95-4.66(\mathrm{~m}, 1 \mathrm{H}, \mathrm{CHO}) .{ }^{13} \mathrm{C} \mathrm{NMR}\left(75 \mathrm{MHz}, \mathrm{DMSO} / \mathrm{CCl}_{4}, 1 / 3\right) \delta 28.0,28.2$, 28.4, 28.6, 32.7, 36.4, 36.6, 38.0, 40.4, 43.9, 44.7, 52.5, 52.8, 53.0, 53.3, 76.2, 76.5, 76.6, 83.2, 82.9, 172.7, 172.5. Anal. calcd. for $\mathrm{C}_{10} \mathrm{H}_{13} \mathrm{~N}_{3} \mathrm{O}_{4}$ : C 50.21; H 5.48; N 17.57\%. Found: C 50.32; H 5.35; N 17.69\%.

General procedure for the synthesis of 3(6)-(4-chloroacetylpiperazin-1-yl)pyridines (7). To a stirred solution of compound $6(5 \mathrm{mmol})$ and pyridine $(6 \mathrm{mmol})$ in absolute benzene $(75 \mathrm{~mL})$ the chloroacetyl chloride $(4.8 \mathrm{~mL}$, $6 \mathrm{mmol}$ ) was added dropwise. The reaction mixture was maintained at $35^{\circ} \mathrm{C}$ for $6 \mathrm{~h}$. After cooling of the reaction mixture at room temperature, the solvent was removed under vacuum and water $(50 \mathrm{~mL})$ was added. The resulting crystals were filtered off, washed with water, dried and recrystallized from ethanol.

3-[4-(Chloroacetyl)piperazin-1-yl]-1-isobutyl-6,7-dihydro-5 $\boldsymbol{H}$-cyclopenta[c]pyridine-4-carbonitrile

(7a). Colorless solid; yield 73\%; mp 118-120 ${ }^{\circ} \mathrm{C}$; IR v/cm ${ }^{-1}$ : 1655 (C=O), 2207 (C=N). ${ }^{1} \mathrm{H}$ NMR (300 MHz, DMSO/CCl $1 / 3) \delta 0.93\left(\mathrm{t}, J=6.6 \mathrm{~Hz}, 6 \mathrm{H}, \mathrm{CH}\left(\mathrm{C}_{3}\right)_{2}\right), 2.07-2.21\left(\mathrm{~m}, 3 \mathrm{H}, 6-\mathrm{CH}_{2}, \underline{\mathrm{C}}\left(\mathrm{CH}_{3}\right)_{2}\right), 2.48\left(\mathrm{~d}, J=7.0 \mathrm{~Hz}, 2 \mathrm{H}, \mathrm{CHC} \underline{H}_{2}\right), 2.84$ $\left(\mathrm{t}, J=7.4 \mathrm{~Hz}, 2 \mathrm{H}, 7-\mathrm{CH}_{2}\right), 3.02\left(\mathrm{t}, J=7.6 \mathrm{~Hz}, 2 \mathrm{H}, 5-\mathrm{CH}_{2}\right), 3.53-3.70\left(\mathrm{~m}, 8 \mathrm{H}, \mathrm{C}_{4} \mathrm{H}_{8} \mathrm{~N}_{2}\right), 4.22\left(\mathrm{~s}, 2 \mathrm{H}, \mathrm{CH}_{2} \mathrm{Cl}\right) .{ }^{13} \mathrm{C} \mathrm{NMR}$ (75 MHz, DMSO/CCl $4,1 / 3) \delta$ 22.14, 23.52, 27.23, 29.34, 32.47, 40.76, 41.08, 44.09, 45.07, 47.71, 48.35, 90.38, 
115.93, 129.54, 158.03, 158.95, 160.11, 164.16. Anal. calcd. for $\mathrm{C}_{19} \mathrm{H}_{25} \mathrm{ClN}_{4} \mathrm{O}$ : C 63.24; $\mathrm{H} 6.98 ; \mathrm{N} 15.53 \%$. Found: C 63.55; H 7.15; N 15.77\%.

6-[4-(Chloroacetyl)piperazin-1-yl]-8-ethyl-3,3-dimethyl-3,4-dihydro-1H-pyrano[3,4-c]pyridine-5-carbonitrile (7b). Colorless solid; yield 70\%; mp 114-116 ${ }^{\circ} \mathrm{C}$; IR v/cm $\mathrm{cm}^{-1}$ : $1659(\mathrm{C}=\mathrm{O}), 2208(\mathrm{C} \equiv \mathrm{N}) .{ }^{1} \mathrm{H} \mathrm{NMR}(300 \mathrm{MHz}$, DMSO/CCl, $1 / 3) \delta 1.24\left(\mathrm{t}, J=7.4 \mathrm{~Hz}, 3 \mathrm{H}, \mathrm{CH}_{2} \mathrm{C}_{3}\right.$ ) $, 1.27\left(\mathrm{~s}, 6 \mathrm{H}, \mathrm{C}\left(\mathrm{CH}_{3}\right)_{2}\right), 2.56\left(\mathrm{q}, J=7.4 \mathrm{~Hz}, 2 \mathrm{H}, \mathrm{C}_{2} \mathrm{CH}_{3}\right), 2.71(\mathrm{~s}$, $\left.2 \mathrm{H}, \mathrm{CH}_{2}\right), 3.57-3.71\left(\mathrm{~m}, 8 \mathrm{H}, \mathrm{C}_{4} \mathrm{H}_{8} \mathrm{~N}_{2}\right), 4.22\left(\mathrm{~s}, 2 \mathrm{H}, \mathrm{CH}_{2} \mathrm{Cl}\right), 4.59\left(\mathrm{~s}, 2 \mathrm{H}, \mathrm{OCH}_{2}\right) .{ }^{13} \mathrm{C} \mathrm{NMR}\left(75 \mathrm{MHz}, \mathrm{DMSO}^{2} \mathrm{CCl}_{4}, 1 / 3\right)$ $\delta$ 10.86, 25.72, 26.43, 37.84, 45.10, 47.45, 48.09, 58.81, 68.97, 93.21, 115.51, 118.85, 147.85, 159.14, 159.35, 164.20. Anal. calcd. for $\mathrm{C}_{19} \mathrm{H}_{25} \mathrm{ClN}_{4} \mathrm{O}_{2}$ : C 60.55; $\mathrm{H}$ 6.69; N 14.87\%. Found: $\mathrm{C} 60.89 ; \mathrm{H} 6.88 ; \mathrm{N} 15.13 \%$.

6-[4-(Chloroacetyl)piperazin-1-yl]-3,3-dimethyl-8-propyl-3,4-dihydro-1H-pyrano[3,4-c]pyridine-5-carbonitrile (7c). Colorless solid; yield 71\%; mp 138-140 ${ }^{\circ} \mathrm{C}$; IR v/cm ${ }^{-1}: 1639$ (C=O), 2209 (C=N). ${ }^{1} \mathrm{H} \mathrm{NMR} \mathrm{(300} \mathrm{MHz,}$ DMSO/CCl $4,1 / 3) \delta 0.99\left(\mathrm{t}, J=7.4 \mathrm{~Hz}, 3 \mathrm{H}, \mathrm{CH}_{2} \mathrm{CH}_{3}\right), 1.27\left(\mathrm{~s}, 6 \mathrm{H}, \mathrm{C}\left(\mathrm{CH}_{3}\right)_{2}\right), 1.66-1.79\left(\mathrm{~m}, 2 \mathrm{H}, \mathrm{CH}_{2} \mathrm{CH}_{3}\right), 2.50(\mathrm{t}, J=$ $\left.7.4 \mathrm{~Hz}, 2 \mathrm{H}, \mathrm{C}_{2} \mathrm{C}_{2} \mathrm{H}_{5}\right), 2.71\left(\mathrm{~s}, 2 \mathrm{H}, \mathrm{CH}_{2}\right), 3.56-3.71\left(\mathrm{~m}, 8 \mathrm{H}, \mathrm{C}_{4} \mathrm{H}_{8} \mathrm{~N}_{2}\right), 4.22\left(\mathrm{~s}, 2 \mathrm{H}, \mathrm{CH}_{2} \mathrm{Cl}\right), 4.59\left(\mathrm{~s}, 2 \mathrm{H}, \mathrm{OCH}_{2}\right) .{ }^{13} \mathrm{C}$ NMR $\left(75 \mathrm{MHz}, \mathrm{DMSO} / \mathrm{CCl}_{4}, 1 / 3\right) \delta 13.51,19.99,25.72,35.18,37.87,40.77,41.13,45.11,47.48,48.13,58.93$, 68.96, 93.34, 115.49, 119.16, 147.95, 158.52, 159.07, 164.20. Anal. calcd. for $\mathrm{C}_{20} \mathrm{H}_{27} \mathrm{ClN}_{4} \mathrm{O}_{2}$ : C 61.45; $\mathrm{H}$ 6.96; N 14.33\%. Found: C 61.83; H 7.19; N 14.62\%.

General procedure for the synthesis of 3(6)-(4-azidoacetylpiperazin-1-yl)pyridines (8). A mixture of compound 7 ( $5 \mathrm{mmol}$ ) and sodium azide $(0.36 \mathrm{~g}, 5.5 \mathrm{mmol})$ in acetone $(30 \mathrm{~mL})$ was refluxed for $15 \mathrm{~h}$. After the filtration the solvent was removed under vacuum and water $(50 \mathrm{~mL})$ was added. The resulting crystals were filtered off, washed with water, dried, and recrystallized from ethanol.

3-[4-(Azidoacetyl)piperazin-1-yl]-1-isobutyl-6,7-dihydro-5H-cyclopenta[c]pyridine-4-carbonitrile

(8a).

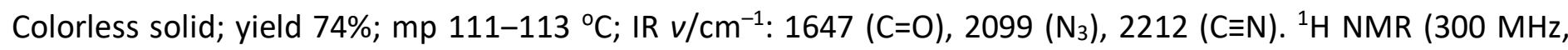
DMSO/CCl, $1 / 3) \delta 0.93\left(\mathrm{t}, J=6.6 \mathrm{~Hz}, 6 \mathrm{H}, \mathrm{CH}\left(\mathrm{C}_{3}\right)_{2}\right), 2.06-2.20\left(\mathrm{~m}, 3 \mathrm{H}, 6-\mathrm{CH}_{2}, \mathrm{C}\left(\mathrm{CH}_{3}\right)_{2}\right), 2.47(\mathrm{~d}, J=7.1 \mathrm{~Hz}, 2 \mathrm{H}$, $\mathrm{CHC}_{2}$ ) , $2.84\left(\mathrm{t}, J=7.4 \mathrm{~Hz}, 2 \mathrm{H}, 7-\mathrm{CH}_{2}\right), 3.01\left(\mathrm{t}, J=7.6 \mathrm{~Hz}, 2 \mathrm{H}, 5-\mathrm{CH}_{2}\right), 3.49-3.72\left(\mathrm{~m}, 8 \mathrm{H}, \mathrm{C}_{4} \mathrm{H}_{8} \mathrm{~N}_{2}\right), 4.04(\mathrm{~s}, 2 \mathrm{H}$, $\left.\mathrm{CH}_{2} \mathrm{~N}_{3}\right) .{ }^{13} \mathrm{C}$ NMR $\left(75 \mathrm{MHz}, \mathrm{DMSO} / \mathrm{CCl}_{4}, 1 / 3\right) \delta 22.16,23.54,27.25,29.36,32.49,40.96,43.81,44.11,47.75$, $48.26,49.57,90.33,115.98,129.52,158.06,158.96,160.14,165.26$. Anal. calcd. for $\mathrm{C}_{19} \mathrm{H}_{25} \mathrm{~N}_{7} \mathrm{O}: \mathrm{C} 62.10 ; \mathrm{H}$ 6.86; N 26.68\%. Found: C 62.42; H 7.03; N 26.93\%.

\section{6-[4-(Azidoacetyl)piperazin-1-yl]-8-ethyl-3,3-dimethyl-3,4-dihydro-1H-pyrano[3,4-c]pyridine-5-carbonitrile}

(8b). Colorless solid; yield 81\%; mp 102-104 ${ }^{\circ} \mathrm{C}$; IR v/cm ${ }^{-1}: 1659$ (C=O), $2107\left(\mathrm{~N}_{3}\right), 2208$ (C三N). ${ }^{1} \mathrm{H} N M R(300$ $\left.\mathrm{MHz}, \mathrm{DMSO} / \mathrm{CCl}_{4}, 1 / 3\right) \delta 1.24\left(\mathrm{t}, J=7.4 \mathrm{~Hz}, 3 \mathrm{H}, \mathrm{CH}_{2} \mathrm{CH}_{3}\right), 1.27\left(\mathrm{~s}, 6 \mathrm{H}, \mathrm{C}\left(\mathrm{CH}_{3}\right)_{2}\right), 2.56\left(\mathrm{q}, J=7.4 \mathrm{~Hz}, 2 \mathrm{H}, \mathrm{CH}_{2} \mathrm{CH}_{3}\right)$, $2.71\left(\mathrm{~s}, 2 \mathrm{H}, \mathrm{CH}_{2}\right), 3.50-3.73\left(\mathrm{~m}, 8 \mathrm{H}, \mathrm{C}_{4} \mathrm{H}_{8} \mathrm{~N}_{2}\right), 4.05\left(\mathrm{~s}, 2 \mathrm{H}, \mathrm{CH}_{2} \mathrm{~N}_{3}\right), 4.59\left(\mathrm{~s}, 2 \mathrm{H}, \mathrm{OCH}_{2}\right) .{ }^{13} \mathrm{C} \mathrm{NMR}(75 \mathrm{MHz}$, $\left.\mathrm{DMSO} \mathrm{CCl}_{4}, 1 / 3\right) \delta 10.86,25.72,26.43,37.84,40.99,43.81,47.49,47.93,49.55,58.81,68.97,93.10,115.53$, $118.80,147.85,159.12,159.35,165.30$. Anal. calcd. for $\mathrm{C}_{19} \mathrm{H}_{25} \mathrm{~N}_{7} \mathrm{O}_{2}$ : C 59.51; H 6.57; N 25.57\%. Found: C 59.86; H 6.78; N 25.84\%.

\section{6-[4-(Azidoacetyl)piperazin-1-yl]-3,3-dimethyl-8-propyl-3,4-dihydro-1H-pyrano[3,4-c]pyridine-5-carbonitrile}

(8c). Colorless solid; yield 83\%; mp 95-97 ${ }^{\circ} \mathrm{C}$; IR v/cm ${ }^{-1}$ : $1641(\mathrm{C}=0), 2103\left(\mathrm{~N}_{3}\right), 2211(\mathrm{C} \equiv \mathrm{N}) .{ }^{1} \mathrm{H} \mathrm{NMR}(300 \mathrm{MHz}$, $\left.\mathrm{DMSO} / \mathrm{CCl}_{4}, 1 / 3\right) \delta 0.98\left(\mathrm{t}, J=7.4 \mathrm{~Hz}, 3 \mathrm{H}, \mathrm{CH}_{2} \mathrm{CH}_{3}\right), 1.27\left(\mathrm{~s}, 6 \mathrm{H}, \mathrm{C}\left(\mathrm{CH}_{3}\right)_{2}\right), 1.66-1.79\left(\mathrm{~m}, 2 \mathrm{H}, \mathrm{C}_{2} \mathrm{CH}_{3}\right), 2.47-2.53$ $\left(\mathrm{m}, 2 \mathrm{H}, \mathrm{CH}_{2} \mathrm{C}_{2} \mathrm{H}_{5}\right), 2.71\left(\mathrm{~s}, 2 \mathrm{H}, \mathrm{CH}_{2}\right), 3.50-3.72\left(\mathrm{~m}, 8 \mathrm{H}, \mathrm{C}_{4} \mathrm{H}_{8} \mathrm{~N}_{2}\right), 4.05\left(\mathrm{~s}, 2 \mathrm{H}, \mathrm{CH}_{2} \mathrm{~N}_{3}\right), 4.59\left(\mathrm{~s}, 2 \mathrm{H}, \mathrm{OCH}_{2}\right) .{ }^{13} \mathrm{CNMR}^{\mathrm{N}}$ (75 MHz, DMSO/CCl $4,1 / 3) \delta 13.52,20.01,25.73,35.19,37.87,41.01,43.84,47.52,47.98,49.57,58.94,68.97$, $93.25,115.53,119.13,147.96,158.53,159.06,165.30$. Anal. calcd. for $\mathrm{C}_{20} \mathrm{H}_{27} \mathrm{~N}_{7} \mathrm{O}_{2}$ : C 60.44; $\mathrm{H} 6.85 ; \mathrm{N} 24.67 \%$. Found: C 60.81; H 7.07; N 24.96\%.

General procedure for the synthesis of propargyl-derivatives (10a-e and 12a,b). To a stirred suspension of compound 9 (11) ( $5 \mathrm{mmol}$ ) and potassium carbonate $(0.76 \mathrm{~g}, 5.5 \mathrm{mmol}$ ) in absolute DMF (30 $\mathrm{mL}$ ) the propargyl bromide $\left(5.5 \mathrm{mmol}\right.$ ) was added dropwise. The reaction mixture was maintained at $50-60{ }^{\circ} \mathrm{C}$ for $5 \mathrm{~h}$. Then the 
reaction mixture was cooled at room temperature, and water was added $(50 \mathrm{~mL})$. The resulting crystals were filtered off, washed with water, dried and recrystallized from ethanol.

1-Isopropyl-3-(prop-2-yn-1-yloxy)-6,7-dihydro-5H-cyclopenta[c]pyridine-4-carbonitrile (10a). Colorless solid; yield 76\%; mp 103-105 ${ }^{\circ} \mathrm{C}$; IR v/cm ${ }^{-1}: 2129(\mathrm{C} \equiv \mathrm{CH}), 2228(\mathrm{C} \equiv \mathrm{N}), 3249(\equiv \mathrm{CH}) .{ }^{1} \mathrm{H} \mathrm{NMR}(300 \mathrm{MHz}, \mathrm{DMSO} \mathrm{CCl}$, $1 / 3) \delta 1.23\left(\mathrm{t}, J=6.8 \mathrm{~Hz}, 6 \mathrm{H}, \mathrm{CH}\left(\underline{\mathrm{H}}_{3}\right)_{2}\right), 2.13-2.24\left(\mathrm{~m}, 2 \mathrm{H}, 6-\mathrm{CH}_{2}\right), 2.89(\mathrm{t}, J=2.4 \mathrm{~Hz}, 1 \mathrm{H}, \equiv \mathrm{CH}), 2.89(\mathrm{t}, J=7.5$ $\left.\mathrm{Hz}, 2 \mathrm{H}, 7-\mathrm{CH}_{2}\right), 2.98-3.07\left(\mathrm{~m}, 3 \mathrm{H}, \mathrm{C} \underline{\mathrm{H}}\left(\mathrm{CH}_{3}\right)_{2}, 5-\mathrm{CH}_{2}\right), 5.03\left(\mathrm{~d}, J=2.4 \mathrm{~Hz}, 2 \mathrm{H}, \mathrm{OCH}_{2}\right) .{ }^{13} \mathrm{C} \mathrm{NMR}(75 \mathrm{MHz}$, DMSO/CCl 4 1/3) $\delta 20.48,23.88,28.64,32.01,32.56,53.45,75.45,78.07,89.90,113.39,129.95,160.32$, 161.07, 162.40. Anal. calcd. for $\mathrm{C}_{15} \mathrm{H}_{16} \mathrm{~N}_{2} \mathrm{O}$ : C 74.97; $\mathrm{H} 6.71 ; \mathrm{N} 11.66 \%$. Found: C 75.31; H 6.90; N $11.92 \%$.

1-Isopropyl-3-(prop-2-yn-1-yloxy)-5,6,7,8-tetrahydroisoquinoline-4-carbonitrile (10b). Colorless solid; yield 84\%; mp 102-104 ${ }^{\circ} \mathrm{C}$; IR v/cm ${ }^{-1}: 2121(\mathrm{C} \equiv \mathrm{CH}), 2220(\mathrm{C} \equiv \mathrm{N}), 3256(\equiv \mathrm{CH}) .{ }^{1} \mathrm{H} \mathrm{NMR}\left(300 \mathrm{MHz}, \mathrm{DMSO} / \mathrm{CCl}_{4}, 1 / 3\right) \delta$ $1.21\left(\mathrm{t}, J=6.7 \mathrm{~Hz}, 6 \mathrm{H}, \mathrm{CH}\left(\mathrm{CH}_{3}\right)_{2}\right), 1.76-1.88\left(\mathrm{~m}, 4 \mathrm{H}, 6,7-\mathrm{CH}_{2}\right), 2.65-2.72\left(\mathrm{~m}, 2 \mathrm{H}, 8-\mathrm{CH}_{2}\right), 2.85-2.90(\mathrm{~m}, 2 \mathrm{H}, 5-$ $\left.\mathrm{CH}_{2}\right), 2.88(\mathrm{t}, J=2.5 \mathrm{~Hz}, 1 \mathrm{H}, \equiv \mathrm{CH}), 3.21\left(\mathrm{t}, J=6.7 \mathrm{~Hz}, 1 \mathrm{H}, \mathrm{C} \underline{\mathrm{H}}\left(\mathrm{CH}_{3}\right)_{2}\right), 5.03\left(\mathrm{~d}, J=2.4 \mathrm{~Hz}, 2 \mathrm{H}, \mathrm{OCH}_{2}\right) .{ }^{13} \mathrm{C} \mathrm{NMR}(75$ $\left.\mathrm{MHz}, \mathrm{DMSO} / \mathrm{CCl}_{4}, 1 / 3\right) \delta 20.77,20.80,21.93,23.82,28.14,30.26,53.02,75.54,78.16,92.80,113.37,122.64$, 152.65, 159.61, 165.91. Anal. calcd. for $\mathrm{C}_{16} \mathrm{H}_{18} \mathrm{~N}_{2} \mathrm{O}$ : C 75.56; H 7.13; N 11.01\%. Found: C 75.92; H 7.33; N 11.29 $\%$.

1-Phenyl-3-(prop-2-yn-1-yloxy)-5,6,7,8-tetrahydroisoquinoline-4-carbonitrile (10c). Colorless solid; yield 62\%; mp 121-123 ${ }^{\circ} \mathrm{C}$; IR v/cm ${ }^{-1}: 2125$ (C三CH), $2218(\mathrm{C} \equiv \mathrm{N}), 3283(\equiv \mathrm{CH}) .{ }^{1} \mathrm{H} \mathrm{NMR}\left(300 \mathrm{MHz}, \mathrm{DMSO} / \mathrm{CCl}_{4}, 1 / 3\right) \delta 1.67-$ $1.76\left(\mathrm{~m}, 2 \mathrm{H}, 6-\mathrm{CH}_{2}\right), 1.84-1.93\left(\mathrm{~m}, 2 \mathrm{H}, 7-\mathrm{CH}_{2}\right), 2.70\left(\mathrm{t}, J=6.2 \mathrm{~Hz}, 2 \mathrm{H}, 8-\mathrm{CH}_{2}\right), 2.97(\mathrm{t}, J=2.5 \mathrm{~Hz}, 1 \mathrm{H}, \equiv \mathrm{CH}), 2.98$

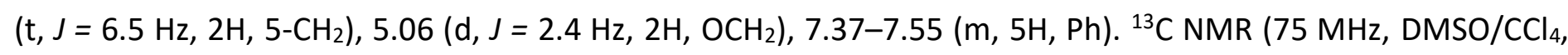
1/3) $\delta$ 20.91, 22.08, 26.63, 28.04, 53.40, 76.06, 78.05, 94.23, 113.21, 124.35, 127.45, 128.17, 128.50, 138.28, 153.75, 158.06, 159.38. Anal. calcd. for $\mathrm{C}_{19} \mathrm{H}_{16} \mathrm{~N}_{2} \mathrm{O}: \mathrm{C} 79.14 ; \mathrm{H} 5.59 ; \mathrm{N} 9.72 \%$. Found: C 79.47; H 5.77; N 9.97\%.

3,3,8-Trimethyl-6-(prop-2-yn-1-yloxy)-3,4-dihydro-1H-pyrano[3,4-c]pyridine-5-carbonitrile (10d). Colorless solid; yield 65\%; mp 128-130 ${ }^{\circ} \mathrm{C}$; IR v/cm ${ }^{-1}: 2133(\mathrm{C} \equiv \mathrm{CH}), 2223(\mathrm{C} \equiv \mathrm{N}), 3259$ (三CH). ${ }^{1} \mathrm{H} \mathrm{NMR}(300 \mathrm{MHz}$, DMSO/CCl $4,1 / 3) \delta 1.28\left(\mathrm{~s}, 6 \mathrm{H}, \mathrm{C}\left(\mathrm{CH}_{3}\right)_{2}\right), 2.37\left(\mathrm{~s}, 3 \mathrm{H}, \mathrm{CH}_{3}\right), 2.76\left(\mathrm{~s}, 2 \mathrm{H}, \mathrm{CH}_{2}\right), 2.94(\mathrm{t}, \mathrm{J}=2.4 \mathrm{~Hz}, 1 \mathrm{H}, \equiv \mathrm{CH}), 4.60(\mathrm{~s}$, $\left.2 \mathrm{H}, \mathrm{OCH}_{2}\right), 5.05\left(\mathrm{~d}, J=2.4 \mathrm{~Hz}, 2 \mathrm{H}, \mathrm{OCH}_{2}\right) .{ }^{13} \mathrm{C} \mathrm{NMR}\left(75 \mathrm{MHz}, \mathrm{DMSO} / \mathrm{CCl}_{4}, 1 / 3\right) \delta 20.69,25.66,37.58,53.35$, $59.15,68.99,76.05,77.81,93.19,112.86,121.45,148.83,155.11,159.98$. Anal. calcd. for $\mathrm{C}_{15} \mathrm{H}_{16} \mathrm{~N}_{2} \mathrm{O}_{2}$ : C 70.29; H 6.29; N 10.93\%. Found: C 70.63; H 6.49; N 11.20\%.

8-Isopropyl-3,3-dimethyl-6-(prop-2-yn-1-yloxy)-3,4-dihydro-1H-pyrano[3,4-c]pyridine-5-carbonitrile (10e). Colorless solid; yield 87\%; mp 147-149 ${ }^{\circ} \mathrm{C}$; IR v/cm ${ }^{-1}: 2133(\mathrm{C} \equiv \mathrm{CH}), 2227(\mathrm{C} \equiv \mathrm{N}), 3277(\equiv \mathrm{CH}) .{ }^{1} \mathrm{H} \mathrm{NMR}(300 \mathrm{MHz}$, $\left.\mathrm{DMSO} / \mathrm{CCl}_{4}, 1 / 3\right) \delta 1.23\left(\mathrm{t}, J=6.6 \mathrm{~Hz}, 6 \mathrm{H}, \mathrm{CH}\left(\mathrm{CH}_{3}\right)_{2}\right), 1.28\left(\mathrm{~s}, 6 \mathrm{H}, \mathrm{C}\left(\mathrm{CH}_{3}\right)_{2}\right), 2.77\left(\mathrm{~s}, 2 \mathrm{H}, \mathrm{CH}_{2}\right), 2.92-2.97(\mathrm{~m}, 2 \mathrm{H}$, $\left.\equiv \mathrm{CH}, \mathrm{CH}\left(\mathrm{CH}_{3}\right)_{2}\right), 4.69\left(\mathrm{~s}, 2 \mathrm{H}, \mathrm{OCH}_{2}\right), 5.05\left(\mathrm{~d}, \mathrm{~J}=2.4 \mathrm{~Hz}, 2 \mathrm{H}, \mathrm{OCH}_{2}\right) .{ }^{13} \mathrm{C} \mathrm{NMR}\left(75 \mathrm{MHz}, \mathrm{DMSO} / \mathrm{CCl}_{4}, 1 / 3\right) \delta 20.69$, 25.68, 30.32, 37.87, 53.31, 58.71, 68.89, 75.87, 77.94, 93.14, 112.95, 119.84, 149.50, 160.31, 163.15. Anal. calcd. for $\mathrm{C}_{17} \mathrm{H}_{20} \mathrm{~N}_{2} \mathrm{O}_{2}$ : C 71.81; H 7.09; N 9.85\%. Found: C 72.13; H 7.26; N 10.09\%.

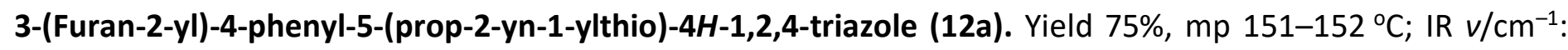
1600, $1613(\mathrm{C}=\mathrm{C}), 1635(\mathrm{C}=\mathrm{N}), 2115(\mathrm{C} \equiv \mathrm{CH}), 3249(\equiv \mathrm{CH}) .{ }^{1} \mathrm{H} \mathrm{NMR}\left(300 \mathrm{MHz}, \mathrm{DMSO} / \mathrm{CCl}_{4}, 1 / 3\right) \delta 2.73(\mathrm{t}, J=2.6$ $\mathrm{Hz}, 1 \mathrm{H}, \equiv \mathrm{CH}), 3.95\left(\mathrm{~d}, J=2.6 \mathrm{~Hz}, 2 \mathrm{H}, \mathrm{SCH}_{2}\right), 6.22\left(\mathrm{~d}, J=3.4 \mathrm{~Hz}, 1 \mathrm{H}_{\text {furyl }}\right), 6.40$ (dd, J = 3.5, $\left.1.8 \mathrm{~Hz}, 1 \mathrm{H}_{\text {furyl }}\right), 7.46-$ $7.33\left(\mathrm{~m}, 2 \mathrm{H}_{\text {arom }}\right), 7.69-7.48\left(\mathrm{~m}, 3 \mathrm{H}_{\text {arom }}, 1 \mathrm{H},=\mathrm{CH}-\mathrm{O}\right) .{ }^{13} \mathrm{C} \mathrm{NMR}\left(75 \mathrm{MHz}, \mathrm{DMSO} / \mathrm{CCl}_{4}, 1 / 3\right) \delta 20.7,73.5,78.0$, $110.7,110.8,127.1,129.3,129.7,133.1,141.0,143.6,147.0,149.6$. Anal. calcd. for $\mathrm{C}_{15} \mathrm{H}_{11} \mathrm{~N}_{3} \mathrm{OS}: \mathrm{C} 64.04 ; \mathrm{H}$ 3.94; N 14.94\%. Found: C 63.91; H 4.011; N 15.13\%.

3-(2-Methoxyphenyl)-4-phenyl-5-(prop-2-yn-1-ylthio)-4H-1,2,4-triazole (12b). Yield 80\%, mp 105-106 ${ }^{\circ} \mathrm{C}$; IR $v / \mathrm{cm}^{-1}:$ 1604, $1618(\mathrm{C}=\mathrm{C}), 1639(\mathrm{C}=\mathrm{N}), 2123(\mathrm{C} \equiv \mathrm{CH}), 3256(\equiv \mathrm{CH}) .{ }^{1} \mathrm{H} \mathrm{NMR}(300 \mathrm{MHz}, \mathrm{DMSO} / \mathrm{CCl} 4,1 / 3) \delta 2.72(\mathrm{t}$, $J=2.6 \mathrm{~Hz}, 1 \mathrm{H}, \equiv \mathrm{CH}), 3.36\left(\mathrm{~s}, J=2.6 \mathrm{~Hz}, 3 \mathrm{H}, \mathrm{OCH}_{3}\right), 3.99\left(\mathrm{~d}, J=2.6 \mathrm{~Hz}, 2 \mathrm{H}, \mathrm{SCH}_{2}\right), 6.79$ (br.d, $J=8.2 \mathrm{~Hz}, 1 \mathrm{H}_{\text {arom }}$ ), 7.01 (td, $\left.J=7.5,0.9 \mathrm{~Hz}, 1 \mathrm{H}_{\text {arom }}\right), 7.21-7.10$ (m, 2H arom), 7.43-7.31 (m, 4Harom), 7.49 (dd, J = 7.5, $1.7 \mathrm{~Hz}, 1 \mathrm{H}_{\text {arom }}$ ). ${ }^{13} \mathrm{C}$ NMR $\left(75 \mathrm{MHz}, \mathrm{DMSO} / \mathrm{CCl}_{4}, 1 / 3\right) \delta 20.4,54.1,78.1,73.3,110.5,115.8,120.0,125.7,128.4,128.5,131.3$, 
133.8, 148.7, 152.9, 156.0. Anal. calcd. for $\mathrm{C}_{18} \mathrm{H}_{15} \mathrm{~N}_{3} \mathrm{OS}$ : C 67.27; $\mathrm{H} 4.70 ; \mathrm{N}$ 13.07\%. Found: $\mathrm{C} 67.39 ; \mathrm{H} 4.56 ; \mathrm{N}$ $13.26 \%$.

General procedure for the synthesis of 1,2,3-triazoles (13a-f). Propargyl derivatives of fused pyridines 10 (5 $\mathrm{mmol})$ and corresponding azides $8(5.5 \mathrm{mmol})$ were suspended in a 1:1 mixture of water and tert-butyl alcohol $(30 \mathrm{~mL})$. Sodium ascorbate $(0.3 \mathrm{mmol}$, of freshly prepared solution in water) was added, followed by copper(II) sulfate pentahydrate $(7.5 \mathrm{mg}, 0.03 \mathrm{mmol}$, in of water). The heterogeneous mixture was stirred vigorously for 5 $\mathrm{h}$ at room temperature and then $10 \mathrm{~h}$ at $60-65^{\circ} \mathrm{C}$. After cooling water was added $(50 \mathrm{~mL})$. The precipitate was collected by filtration, washed with water and recrystallized from a mixture of ethanol-chloroforme (1:3).

3-[(1-\{2-[4-(4-Cyano-1-isobutyl-6,7-dihydro-5H-cyclopenta[c]pyridin-3-yl)piperazin-1-yl]-2-oxoethyl\}-1H1,2,3-triazol-4-yl)methoxy]-1-isopropyl-6,7-dihydro-5H-cyclopenta[c]pyridine-4-carbonitrile (13a). Colorless solid; yield 72\%; mp 162-164 ${ }^{\circ} \mathrm{C}$; IR v/cm ${ }^{-1}$ : 1662 (C=O), 2211, 2225 (C=N). ${ }^{1} \mathrm{H}$ NMR (300 MHz, DMSO/CCl, $\left.1 / 3\right)$ $\delta 0.94\left(\mathrm{~d}, J=6.5 \mathrm{~Hz}, 6 \mathrm{H}, \mathrm{CH}_{2} \mathrm{CH}\left(\mathrm{CH}_{3}\right)_{2}\right), 1.27\left(\mathrm{~d}, J=6.7 \mathrm{~Hz}, 6 \mathrm{H}, \mathrm{CH}\left(\mathrm{C}_{3}\right)_{2}\right), 2.08-2.24\left(\mathrm{~m}, 5 \mathrm{H}, 6,6^{\prime}-\mathrm{CH}_{2}\right.$, $\left.\mathrm{CH}_{2} \mathrm{C} \underline{\mathrm{H}}\left(\mathrm{CH}_{3}\right)_{2}\right), 2.49\left(\mathrm{~d}, J=6.5 \mathrm{~Hz}, 2 \mathrm{H}, \mathrm{CH}_{2} \mathrm{CH}\right), 2.81-2.96\left(\mathrm{~m}, 4 \mathrm{H}, 7,7^{\prime}-\mathrm{CH}_{2}\right), 2.99-3.10\left(\mathrm{~m}, 5 \mathrm{H}, 5,5^{\prime}-\mathrm{CH}_{2}\right.$, $\left.\mathrm{C} \underline{\mathrm{H}}\left(\mathrm{CH}_{3}\right)_{2}\right), 3.56-3.75\left(\mathrm{~m}, 8 \mathrm{H}, \mathrm{C}_{4} \mathrm{H}_{8} \mathrm{~N}_{2}\right), 5.47\left(\mathrm{~s}, 2 \mathrm{H}, \mathrm{NCH}_{2}\right), 5.55\left(\mathrm{~s}, 2 \mathrm{H}, \mathrm{OCH}_{2}\right), 7.93\left(\mathrm{~s}, 1 \mathrm{H}, \mathrm{CH}_{\text {triazole }}\right) .{ }^{13} \mathrm{C} \mathrm{NMR}(75$ $\left.\mathrm{MHz}, \mathrm{DMSO} / \mathrm{CCl}_{4}, 1 / 3\right) \delta 20.60,22.16,23.53,23.89,27.24,28.68,29.35,32.00,32.49,32.69,41.16,43.94$, $44.09,47.65,48.22,50.32,59.63,89.75,90.23,113.81,116.01,125.19,129.46,129.58,141.79,158.05$, $158.93,160.14,161.01,161.22,162.61,163.70$. Anal. calcd. for $\mathrm{C}_{34} \mathrm{H}_{41} \mathrm{~N}_{9} \mathrm{O}_{2}$ : C 67.19; $\mathrm{H} 6.80 ; \mathrm{N} 20.74 \%$. Found: C 67.57; H 7.02; N 21.03\%.

6-\{4-[(4-\{[(4-Cyano-1-isopropyl-6,7-dihydro-5H-cyclopenta[c]pyridin-3-yl)oxy]methyl\}-1H-1,2,3-triazol-1yl)acetyl]piperazin-1-yl\}-8-ethyl-3,3-dimethyl-3,4-dihydro-1H-pyrano[3,4-c]pyridine-5-carbonitrile (13b).

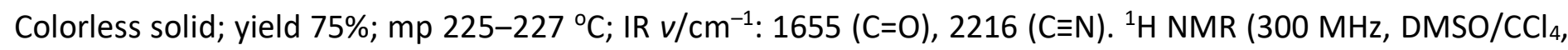
$1 / 3) \delta 1.25\left(\mathrm{t}, J=7.5 \mathrm{~Hz}, 3 \mathrm{H}, \mathrm{CH}_{2} \mathrm{C}_{3}\right), 1.27\left(\mathrm{~d}, J=6.7 \mathrm{~Hz}, 6 \mathrm{H}, \mathrm{CH}\left(\mathrm{CH}_{3}\right)_{2}\right), 1.28\left(\mathrm{~s}, 6 \mathrm{H}, \mathrm{C}\left(\mathrm{CH}_{3}\right)_{2}\right), 2.13-2.24(\mathrm{~m}, 2 \mathrm{H}$, $6^{\prime}-\mathrm{CH}_{2}$ ), 2.57 (q, J = 7.4 Hz, 2H, $\left.\mathrm{CH}_{2} \mathrm{CH}_{3}\right), 2.72\left(\mathrm{~s}, 2 \mathrm{H}, 4-\mathrm{CH}_{2}\right), 2.90\left(\mathrm{t}, J=7.4 \mathrm{~Hz}, 2 \mathrm{H}, 7^{\prime}-\mathrm{CH}_{2}\right), 3.03(\mathrm{t}, J=7.4 \mathrm{~Hz}$, $\left.2 \mathrm{H}, 5^{\prime}-\mathrm{CH}_{2}\right), 3.05\left(\mathrm{sp}, J=6.7 \mathrm{~Hz}, 1 \mathrm{H}, \mathrm{C} \underline{\mathrm{H}}\left(\mathrm{CH}_{3}\right)_{2}\right), 3.61-3.76\left(\mathrm{~m}, 8 \mathrm{H}, \mathrm{C}_{4} \mathrm{H}_{8} \mathrm{~N}_{2}\right), 4.60\left(\mathrm{~s}, 2 \mathrm{H}, 1-\mathrm{CH}_{2}\right), 5.48(\mathrm{~s}, 2 \mathrm{H}$, $\mathrm{NCH}_{2}$ ), 5.55 (s, 2H, OCH ), 7.93 (s, 1H, CH triazole). $\left.{ }^{13} \mathrm{C} \mathrm{NMR} \mathrm{(75} \mathrm{MHz,} \mathrm{DMSO/CCl}, 1 / 3\right) \delta 10.88,20.61,23.90$, 25.75, 26.45, 28.68, 32.01, 32.70, 37.86, 41.19, 43.96, 47.41, 47.93, 50.35, 58.83, 59.66, 68.98, 89.76, 93.05, $113.81,115.60,118.77,125.18,129.58,141.80,147.86,159.13,159.39,161.01,161.23,162.61,163.78$. Anal. calcd. for $\mathrm{C}_{34} \mathrm{H}_{41} \mathrm{~N}_{9} \mathrm{O}_{3}$ : C 65.47; $\mathrm{H} 6.63 ; \mathrm{N} 20.21 \%$. Found: C 65.83; H 6.82; N 20.47\%.

6-\{4-[(4-\{[(4-Cyano-1-isopropyl-5,6,7,8-tetrahydroisoquinolin-3-yl)oxy]methyl\}-1H-1,2,3-triazol-1yl)acetyl]piperazin-1-yl\}-8-ethyl-3,3-dimethyl-3,4-dihydro-1H-pyrano[3,4-c]pyridine-5-carbonitrile

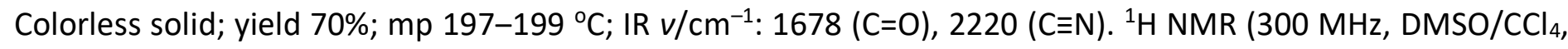
$1 / 3) \delta 1.25\left(\mathrm{t}, J=7.4 \mathrm{~Hz}, 3 \mathrm{H}, \mathrm{CH}_{2} \mathrm{CH}_{3}\right), 1.25\left(\mathrm{~d}, J=6.6 \mathrm{~Hz}, 6 \mathrm{H}, \mathrm{CH}\left(\mathrm{CH}_{3}\right)_{2}\right), 1.28\left(\mathrm{~s}, 6 \mathrm{H}, \mathrm{C}\left(\mathrm{CH}_{3}\right)_{2}\right), 1.77-1.88(\mathrm{~m}, 4 \mathrm{H}$, $\left.6^{\prime}, 7^{\prime}-\mathrm{CH}_{2}\right), 2.57\left(\mathrm{t}, J=7.4 \mathrm{~Hz}, 2 \mathrm{H}, \mathrm{CH}_{2} \mathrm{CH}_{3}\right), 2.66-2.73\left(\mathrm{~m}, 2 \mathrm{H}, 8^{\prime}-\mathrm{CH}_{2}\right), 2.72\left(\mathrm{~s}, 2 \mathrm{H}, 4-\mathrm{CH}_{2}\right), 2.84-2.89\left(\mathrm{~m}, 2 \mathrm{H}, 5^{\prime}-\right.$ $\left.\mathrm{CH}_{2}\right), 3.22\left(\mathrm{sp}, J=6.6 \mathrm{~Hz}, 1 \mathrm{H}, \mathrm{C} \underline{\mathrm{H}}\left(\mathrm{CH}_{3}\right)_{2}\right), 3.61-3.76\left(\mathrm{~m}, 8 \mathrm{H}, \mathrm{C}_{4} \mathrm{H}_{8} \mathrm{~N}_{2}\right), 4.60\left(\mathrm{~s}, 2 \mathrm{H}, 1-\mathrm{CH}_{2}\right), 5.48(\mathrm{~s}, 2 \mathrm{H}, \mathrm{NCH}), 5.55$

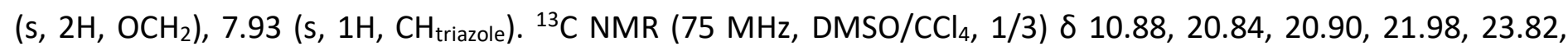
25.75, 26.45, 28.12, 30.36, 37.87, 41.20, 43.96, 47.41, 47.91, 50.34, 58.82, 59.19, 68.98, 92.70, 93.04, 113.71, $115.60,118.77,122.25,125.13,141.85,147.86,152.53,159.13,159.39,160.48,163.78,166.05$. Anal. calcd. for $\mathrm{C}_{35} \mathrm{H}_{43} \mathrm{~N}_{9} \mathrm{O}_{3}$ : C 65.91; H 6.80; N 19.77\%. Found: C 66.30; H 7.03; N 20.05\%.

3-[(1-\{2-[4-(4-Cyano-1-isobutyl-6,7-dihydro-5H-cyclopenta[c]pyridin-3-yl)piperazin-1-yl]-2-oxoethyl\}-1H1,2,3-triazol-4-yl)methoxy]-1-phenyl-5,6,7,8-tetrahydroisoquinoline-4-carbonitrile (13d). Colorless solid;

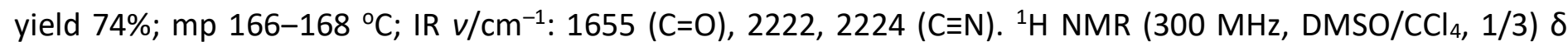
$0.94\left(\mathrm{t}, J=6.6 \mathrm{~Hz}, 6 \mathrm{H}, \mathrm{CH}\left(\mathrm{CH}_{3}\right)_{2}\right), 1.67-1.76\left(\mathrm{~m}, 2 \mathrm{H}, 6-\mathrm{CH}_{2}\right), 1.83-1.93\left(\mathrm{~m}, 2 \mathrm{H}, 7-\mathrm{CH}_{2}\right), 2.07-2.22\left(\mathrm{~m}, 3 \mathrm{H}, 6^{\prime}-\mathrm{CH}_{2}\right.$, $\left.\left.\mathrm{C} \underline{\mathrm{H}}\left(\mathrm{CH}_{3}\right)_{2}\right)\right), 2.48\left(\mathrm{~d}, J=7.0 \mathrm{~Hz}, 2 \mathrm{H}, \mathrm{CHC}_{2}\right), 2.69\left(\mathrm{t}, J=6.1 \mathrm{~Hz}, 2 \mathrm{H}, 8-\mathrm{CH}_{2}\right), 2.85\left(\mathrm{t}, J=7.4 \mathrm{~Hz}, 2 \mathrm{H}, 7^{\prime}-\mathrm{CH}_{2}\right), 2.94-$ $3.06\left(\mathrm{~m}, 4 \mathrm{H}, 5,5^{\prime}-\mathrm{CH}_{2}\right), 3.56-3.76\left(\mathrm{~m}, 8 \mathrm{H}, \mathrm{C}_{4} \mathrm{H}_{8} \mathrm{~N}_{2}\right), 5.48\left(\mathrm{~s}, 2 \mathrm{H}, \mathrm{NCH}_{2}\right), 5.56\left(\mathrm{~s}, 2 \mathrm{H}, \mathrm{OCH}_{2}\right), 7.38-7.57(\mathrm{~m}, 5 \mathrm{H}, \mathrm{Ph})$, 
\left.7.93 (s, 1H, CH${\text { triazole). }{ }^{13} \mathrm{C} \text { NMR (75 MHz, DMSO/CCl}}_{4}, 1 / 3\right) \delta 20.94,22.10,22.14,23.52,26.57,27.22,27.99$, 29.33, 32.47, 41.14, 43.93, 44.09, 47.62, 47.64, 50.32, 59.46, 90.20, 94.10, 113.54, 116.01, 123.91, 125.50, $127.51,128.08,128.49,129.45,138.47,153.61,158.05,158.33,158.91,160.13,160.22,163.72$. Anal. calcd. for $\mathrm{C}_{38} \mathrm{H}_{41} \mathrm{~N}_{9} \mathrm{O}_{2}$ : C 69.60; H 6.30; N 19.22\%. Found: C 69.91; H 6.48; N 19.47\%.

6-[(1-\{2-[4-(5-Cyano-3,3-dimethyl-8-propyl-3,4-dihydro-1H-pyrano[3,4-c]pyridin-6-yl)piperazin-1-yl]-2oxoethyl\}-1H-1,2,3-triazol-4-yl)methoxy]-3,3,8-trimethyl-3,4-dihydro-1H-pyrano[3,4-c]pyridine-5-

carbonitrile (13e). Colorless solid; yield 71\%; mp 234-236 ${ }^{\circ} \mathrm{C} ; \mathrm{IR} \mathrm{v} / \mathrm{cm}^{-1}: 1664$ (C=O), 2206, 2228 (C三N). ${ }^{1} \mathrm{H}$ NMR $\left(300 \mathrm{MHz}, \mathrm{DMSO} / \mathrm{CCl}_{4}, 1 / 3\right) \delta 1.00\left(\mathrm{t}, J=7.4 \mathrm{~Hz}, 3 \mathrm{H}, \mathrm{CH}_{2} \mathrm{CH}_{3}\right), 1.28\left(\mathrm{~s}, 12 \mathrm{H}, 2 \mathrm{C}\left(\mathrm{CH}_{3}\right)_{2}\right), 1.67-1.80(\mathrm{~m}, 2 \mathrm{H}$, $\left.\mathrm{C}_{2} \mathrm{CH}_{3}\right), 2.41\left(\mathrm{~s}, 3 \mathrm{H}, \mathrm{CH}_{3}\right), 2.48-2.54\left(\mathrm{~m}, 2 \mathrm{H}, \underline{\mathrm{CH}}_{2} \mathrm{C}_{2} \mathrm{H}_{5}\right), 2.72\left(\mathrm{~s}, 2 \mathrm{H}, 4-\mathrm{CH}_{2}\right), 2.75\left(\mathrm{~s}, 2 \mathrm{H}, 4^{\prime}-\mathrm{CH}_{2}\right), 3.59-3.76(\mathrm{~m}$, $\left.8 \mathrm{H}, \mathrm{C}_{4} \mathrm{H}_{8} \mathrm{~N}_{2}\right), 4.60\left(\mathrm{~s}, 2 \mathrm{H}, 1-\mathrm{CH}_{2}\right), 4.61\left(\mathrm{~s}, 2 \mathrm{H}, 1^{\prime}-\mathrm{CH}_{2}\right), 5.48\left(\mathrm{~s}, 2 \mathrm{H}, \mathrm{NCH}_{2}\right), 5.55\left(\mathrm{~s}, 2 \mathrm{H}, \mathrm{OCH}_{2}\right), 7.97(\mathrm{~s}, 1 \mathrm{H}$, $\mathrm{CH}_{\text {triazole). }}{ }^{13} \mathrm{C}$ NMR $\left(75 \mathrm{MHz}, \mathrm{DMSO} / \mathrm{CCl}_{4}, 1 / 3\right) \delta 13.53,20.01,20.80,25.68,25.74,35.20,37.57,37.88,41.20$, 41.22, 43.95, 47.40, 47.96, 50.35, 58.95, 59.18, 59.53, 68.97, 69.00, 92.97, 93.16, 113.27, 115.58, 119.08, $121.00,125.66,141.53,147.96,148.74,155.30,158.55,159.05,160.84,163.79$. Anal. calcd. for $\mathrm{C}_{35} \mathrm{H}_{43} \mathrm{~N}_{9} \mathrm{O}_{4}: \mathrm{C}$ 64.30; H 6.63; N 19.28\%. Found: C 64.65; H 6.86; N $19.55 \%$.

6-[(1-\{2-[4-(5-Cyano-8-ethyl-3,3-dimethyl-3,4-dihydro-1H-pyrano[3,4-c]pyridin-6-yl)piperazin-1-yl]-2oxoethyl\}-1H-1,2,3-triazol-4-yl)methoxy]-8-isopropyl-3,3-dimethyl-3,4-dihydro-1H-pyrano[3,4-c]pyridine-5carbonitrile (13f). Colorless solid; yield 76\%; mp 247-249 ${ }^{\circ} \mathrm{C}$; IR v/cm ${ }^{-1}: 1660$ (C=O), 2214, 2234 (C三N). ${ }^{1} \mathrm{H} N M R$ (300 MHz, DMSO/CCl, $1 / 3) \delta 1.25\left(\mathrm{t}, J=7.4 \mathrm{~Hz}, 3 \mathrm{H}, \mathrm{CH}_{2} \mathrm{CH}_{3}\right), 1.27\left(\mathrm{~d}, J=6.7 \mathrm{~Hz}, 6 \mathrm{H}, \mathrm{CH}\left(\mathrm{C}_{3}\right)_{2}\right), 1.28(\mathrm{~s}, 12 \mathrm{H}$, $\left.2 \mathrm{C}\left(\mathrm{CH}_{3}\right)_{2}\right), 2.57\left(\mathrm{q}, J=7.4 \mathrm{~Hz}, 2 \mathrm{H}, \mathrm{CH}_{2} \mathrm{CH}_{3}\right), 2.72\left(\mathrm{~s}, 2 \mathrm{H}, 4-\mathrm{CH}_{2}\right), 2.76\left(\mathrm{~s}, 2 \mathrm{H}, 4^{\prime}-\mathrm{CH}_{2}\right), 2.95(\mathrm{sp}, J=6.7 \mathrm{~Hz}, 1 \mathrm{H}$, $\left.\mathrm{C} \underline{\mathrm{H}}\left(\mathrm{CH}_{3}\right)_{2}\right), 3.61-3.76\left(\mathrm{~m}, 8 \mathrm{H}, \mathrm{C}_{4} \mathrm{H}_{8} \mathrm{~N}_{2}\right), 4.60\left(\mathrm{~s}, 2 \mathrm{H}, 1-\mathrm{CH}_{2}\right), 4.69\left(\mathrm{~s}, 2 \mathrm{H}, 1^{\prime}-\mathrm{CH}_{2}\right), 5.48\left(\mathrm{~s}, 2 \mathrm{H}, \mathrm{NCH}_{2}\right), 5.57(\mathrm{~s}, 2 \mathrm{H}$,

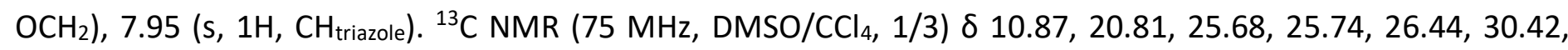
$37.86,41.18,43.95,47.40,47.91,50.35,58.74,58.78,58.82,59.42,68.87,68.97,93.04,93.07,113.29,115.59$, $118.77,119.42,125.26,141.61,147.86,149.37,159.12,159.38,161.18,163.28,163.76$. Anal. calcd. for $\mathrm{C}_{36} \mathrm{H}_{45} \mathrm{~N}_{9} \mathrm{O}_{4}$ : C 64.75; $\mathrm{H} 6.79 ; \mathrm{N} 18.88 \%$. Found: C 65.08; H 6.98; N 19.12\%.

General procedure for the synthesis of 1,2,3-triazoles (14-17). A $5 \mathrm{~mL}$ vial with a screw cup was charged with corresponding azide $3 / 5(0.5 \mathrm{mmol}), \mathrm{MeCN}(4.5 \mathrm{~mL})$, Cul $(0.0048 \mathrm{~g}, 0.025 \mathrm{mmol}, 5 \mathrm{~mol} \%), \mathrm{Et}_{3} \mathrm{~N}(0.061 \mathrm{~g}, 0.6$ $\mathrm{mmol}), \mathrm{Na}_{2} \mathrm{SO}_{3}(0.0063 \mathrm{~g}, 0.05 \mathrm{mmol})$ and propargyl derivative 10/12 (0.55 mmol) and heated for $5 \mathrm{~h}$ at 60-65 ${ }^{\circ} \mathrm{C}$. Reaction mixture was poured into $0.1 \mathrm{M} \mathrm{HCl}(30 \mathrm{~mL})$ and extracted with $\mathrm{DCM}(3 \times 10 \mathrm{~mL})$. Combined extracts were dried over $\mathrm{Na}_{2} \mathrm{SO}_{4}$, volatiles were evaporated and the residue was purified via column chromatography on silica gel mixture of DCM-MeOH (30:1) as an eluent.

3-\{[4-(\{[5-(furan-2-yl)-4-phenyl-4H-1,2,4-triazol-3-yl]thio\}methyl)-1H-1,2,3-triazol-1-yl]methyl\}-8-methyl-2,7dioxaspiro[4.4]nonane-1,6-dione (14). Yield 68\%, mp 180-181 ${ }^{\circ} \mathrm{C} . \mathrm{IR} \mathrm{v} / \mathrm{cm}^{-1}: 1601,1615$ (C=C), 1772,1792 (C=O). ${ }^{1} \mathrm{H}$ NMR $\left(300 \mathrm{MHz}, \mathrm{DMSO} / \mathrm{CCl}_{4}-1 / 3\right) \delta 1.36-1.49\left(\mathrm{~m}, 3 \mathrm{H}, \mathrm{CH}_{3}\right), 1.86-2.11\left(\mathrm{~m}, 1 \mathrm{H}^{\mathrm{a}}, \mathrm{CH}_{2}\right.$ in cycle), 2.12$2.44\left(\mathrm{~m}, 1 \mathrm{H}^{\mathrm{b}}, \mathrm{CH}_{2}\right.$ in cycle), 2.52-2.70 (m, $1 \mathrm{H}^{\mathrm{a}}, \mathrm{CH}_{2}$ in cycle), 2.78-2.87 (m, $1 \mathrm{H}^{\mathrm{b}}, \mathrm{CH}_{2}$ in cycle), $4.46\left(\mathrm{~s}, 2 \mathrm{H}, \mathrm{SCH}_{2}\right)$, 4.64-4.79 (m, 2H, NCH$)$, 4.79-4.92 (m, $\left.1 \mathrm{H}, \mathrm{CH}_{3} \underline{\mathrm{CHO}}\right), 4.93-5.22\left(\mathrm{~m}, 1 \mathrm{H}, \mathrm{NCH}_{2} \underline{\mathrm{CHO}}\right), 6.18(\mathrm{dd}, \mathrm{J}=3.5,0.6 \mathrm{~Hz}$, $\left.1 \mathrm{H}_{\text {furyl }}\right), 6.39\left(\mathrm{dd}, J=3.5,1.8 \mathrm{~Hz}, 1 \mathrm{H}_{\text {furyl }}\right), 7.33-7.41\left(\mathrm{~m}, 2 \mathrm{H}_{\text {arom }}\right), 7.51-7.59\left(\mathrm{~m}, 3 \mathrm{H}_{\text {arom }}, 1 \mathrm{H},=\mathrm{CH}-0\right), 7.97-8.06(\mathrm{~m}$, $1 \mathrm{H},=\mathrm{CH}-\mathrm{N}) .{ }^{13} \mathrm{C}$ NMR $(75 \mathrm{MHz}$, DMSO/CCl, $1 / 3) \delta$ 20.2, 26.5, 34.0, 51.9, 52.5, 75.1, 76.3, 110.6, 110.8, 127.1, 127.7, 129.3, 129.7, 133.2, 143.5 172.3, 172.7. Anal. calcd. for $\mathrm{C}_{24} \mathrm{H}_{22} \mathrm{~N}_{6} \mathrm{O}_{5} \mathrm{~S}$ : C 56.91; $\mathrm{H} 4.38 ; \mathrm{N} 16.59 \%$. Found: C 57.16; H 4.19; N 16.76\%.

(R)-5-\{3-[4-(\{[5-(furan-2-yl)-4-phenyl-4H-1,2,4-triazol-3-yl]thio\}methyl)-1H-1,2,3-triazol-1-yl]prop-1-en-2-yl\}-

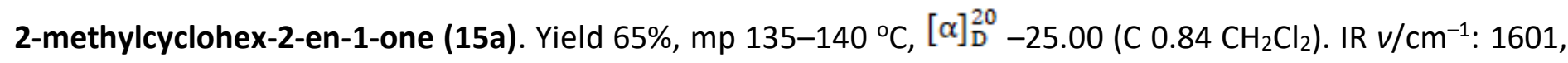
1615, $1635(\mathrm{C}=\mathrm{C}), 1645(\mathrm{C}=\mathrm{N}), 1705(\mathrm{C}=0) .{ }^{1} \mathrm{H} \mathrm{NMR}\left(300 \mathrm{MHz}, \mathrm{DMSO} / \mathrm{CCl}_{4}, 1 / 3\right) \delta 1.68\left(\mathrm{br} . \mathrm{s}, 3 \mathrm{H}, \mathrm{CH}_{3}\right), 2.52-$ $2.16\left(\mathrm{~m}, 4 \mathrm{H}, \mathrm{CH}_{2}\right.$ in carbocycle), $2.58\left(\mathrm{~m}, 1 \mathrm{H}, \mathrm{CH}\right.$ in carbocycle), 4.43 (br.s, $\left.2 \mathrm{H}, \mathrm{CH}_{2} \mathrm{~S}\right), 4.91$ (br.s, $\left.1 \mathrm{H}^{\mathrm{b}}, \mathrm{CH}_{2} \mathrm{~N}\right)$, 
5.01 (br.s, $1 \mathrm{H}^{\mathrm{b}},=\mathrm{CH}_{2}, 1 \mathrm{H}^{\mathrm{a}}, \mathrm{CH}_{2} \mathrm{~N}$ ), 5.05 (br.s, $1 \mathrm{H}^{\mathrm{a}},=\mathrm{CH}_{2}$ ), 6.18 (br.d, $J=3.2 \mathrm{~Hz}, 1 \mathrm{H}_{\text {furyl }}$ ), 6.39 (br.dd, $J=2.9,1.3$ $\left.\mathrm{Hz}, 1 \mathrm{H}_{\text {furyl }}\right), 6.62-6.77(\mathrm{~m}, 1 \mathrm{H}, \underline{\mathrm{CH}}=\mathrm{C}-\mathrm{C}=0), 7.23-7.40\left(\mathrm{~m}, 2 \mathrm{H}_{\text {arom }}\right), 7.48-7.61\left(\mathrm{~m}, 3 \mathrm{H}_{\text {arom }}, 1 \mathrm{H}=\mathrm{CHO}\right), 7.99$ (br.s, J = $4.8 \mathrm{~Hz}, 1 \mathrm{H},=\mathrm{CHN}) .{ }^{13} \mathrm{C} \mathrm{NMR}\left(75 \mathrm{MHz}, \mathrm{DMSO} / \mathrm{CCl}_{4}, 1 / 3\right) \delta 14.9,30.2,37.7,41.8,52.6,110.3,110.7,113.4$, 129.1, 129.5, 126.9, 133.2, 134.2, 141.0, 142.9, 143.4, 145.1, 196.4. Anal. calcd. for $\mathrm{C}_{25} \mathrm{H}_{24} \mathrm{~N}_{6} \mathrm{O}_{2} \mathrm{~S}: \mathrm{C} 63.54 ; \mathrm{H}$ 5.12; N 17.78\%. Found: C 63.35; H 5.01; N 17.96\%.

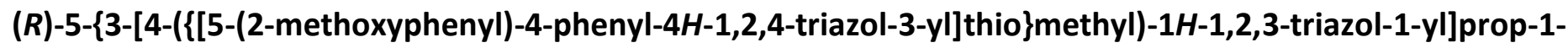
en-2-yl\}-2-methylcyclohex-2-en-1-one (15b). Yield 64\%, mp 69-72 ${ }^{\circ} \mathrm{C},[\alpha]_{\mathrm{D}}^{20}-22.00\left(\mathrm{C} 0.92 \mathrm{CH}_{2} \mathrm{Cl}_{2}\right) . \mathrm{IR} \mathrm{v} \mathrm{cm}^{-1}$ : 1600, 1612, 1640 (C=C), 1645 (C=N), 1706 (C=O). ${ }^{1} \mathrm{H}$ NMR (300 MHz, DMSO/CCl $\left.4,1 / 3\right) \delta 1.70$ (br.s, 3H, CH ${ }_{3} \mathrm{C}-$ $\mathrm{C}=0)$. 2.14-2.41 (m, $2 \mathrm{H}, \mathrm{CH}_{2}$ in carbocycle), 2.42-2.55 (m, 2H, $\mathrm{CH}_{2}$ in carbocycle), 2.56-2.77 (m, $1 \mathrm{H}, \mathrm{CH}$ in carbocycle), $3.34\left(\mathrm{~s}, 3 \mathrm{H}, \mathrm{OCH}_{3}\right), 4.47\left(\mathrm{~s}, 2 \mathrm{H}, \mathrm{CH}_{2} \mathrm{~S}\right), 4.91\left(\mathrm{~s}, 1 \mathrm{H}^{\mathrm{b}}, \mathrm{H}_{2} \mathrm{C}=\right), 5.02\left(\mathrm{~s}, 2 \mathrm{H}, \mathrm{CH}_{2} \mathrm{~N}\right), 5.07\left(\mathrm{~s}, 1 \mathrm{H}^{\mathrm{a}}, \mathrm{H}_{2} \mathrm{C}=\right)$ ), 6.65-6.74 (m, 1H, $\underline{\mathrm{HC}}=\mathrm{C}-\mathrm{C}=\mathrm{O}), 6.78$ (br.d, $\left.J=8.4 \mathrm{~Hz}, 1 \mathrm{H}_{\text {arom }}\right), 7.01$ (br.t, $\left.J=7.1 \mathrm{~Hz}, 1 \mathrm{H}_{\text {arom }}\right), 7.06-7.21$ (m, $\left.2 \mathrm{H}_{\text {arom }}\right), 7.32-7.44\left(\mathrm{~m}, 4 \mathrm{H}_{\text {arom }}\right), 7.49$ (br.d, $\left.J=7.1 \mathrm{~Hz}, 1 \mathrm{H}_{\text {arom }}\right), 7.97$ (s, $\left.1 \mathrm{H}=\mathrm{CHN}\right) .{ }^{13} \mathrm{C} \mathrm{NMR}\left(75 \mathrm{MHz}, \mathrm{DMSO} \mathrm{CCl}_{4}\right.$, 1/3) $\delta 15.1,26.3,30.4,37.9,42.0,52.5,54.1,110.5,113.4,115.9,120.0,125.6,127.6,128.3,128.4,131.2$, $131.2,133.9,134.3,143.1,145.4,155.9,196.6$. Anal. calcd. for $\mathrm{C}_{28} \mathrm{H}_{28} \mathrm{~N}_{6} \mathrm{O}_{2} \mathrm{~S}$ : C 65.60; $\mathrm{H} 5.51 ; \mathrm{N} 16.39 \%$. Found: C 65.76; H 5.33; N 16.57\%.

6-(\{1-[(8,8-Dimethyl-1,6-dioxo-2,7-dioxaspiro[4-4]nonan-3-yl)methyl]-1H-1,2,3-triazol-4-yl\}methoxy)-3,3,8trimethyl-3,4-dihydro-1H-pyrano[3,4-c]pyridine-5-carbonitrile (16a). Yield 73\%, mp 172-175 ${ }^{\circ} \mathrm{C} . \mathrm{IR} \mathrm{v} / \mathrm{cm}^{-1}$ : 1772, 1792 (C=O), 2203 (C=N). ${ }^{1} \mathrm{H}$ NMR (300 MHz, DMSO/CCl, $\left.1 / 3\right) \delta 1.27\left(\mathrm{~s}, 6 \mathrm{H}, \mathrm{OC}\left(\mathrm{CH}_{3}\right)_{2}\right), 1.50-1.40(\mathrm{~m}, 3 \mathrm{H}$, on lactone), $1.60-1.50\left(\mathrm{~m}, 3 \mathrm{H}\right.$, on lactone), 2.38-2.04 (m, 2H, $\mathrm{CH}_{2}$ in lactone), $2.40\left(\mathrm{~s}, 3 \mathrm{H}, \mathrm{N}=\mathrm{C}-\mathrm{CH}_{3}\right), 2.70-2.44$ (m, $2 \mathrm{H}, \mathrm{CH}_{2}$ in lactone), $2.74\left(\mathrm{~s}, 2 \mathrm{H}, \mathrm{CH}_{2}\right.$ in pyran), 4.61 (s, 2H, $\mathrm{CH}_{2} \mathrm{O}$ in cycle), 4.85-4.67 (m, 2H, $\left.\mathrm{CH}_{2} \mathrm{~N}\right), 5.21-$

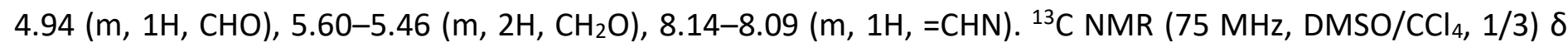
20.8, 25.6, 25.7, 25.7, 25.8, 28.0, 28.2, 28.4, 28.6, 36.4, 36.7, 37.6, 43.6, 44.5, 51.8, 52.9, 53.2, 59.2, 69.0, 75.8, 76.3, 83.1, 83.2, 93.0, 113.2, 121.1, 121.1, 125.1, 125.2, 141.9, 142.2, 148.7, 155.3, 160.7, 172.4, 172.6. Anal. calcd. for $\mathrm{C}_{25} \mathrm{H}_{31} \mathrm{~N}_{5} \mathrm{O}_{6}$ : C 60.35; H 6.28; N 14.08\%. Found: C 60.52; H 6.10; N 14.15\%.

3-(\{1-[(6,6-Dimethyl-3,8-dioxo-2,7-dioxaspiro[4.4]nonan-1-yl)methyl]-1H-1,2,3-triazol-4-yl\}methoxy)-1isopropyl-6,7-dihydro-5H-cyclopenta[c]pyridine-4-carbonitrile (16b). Yield 76\%, mp 64-65 ${ }^{\circ} \mathrm{C} . \quad \mathrm{IR} v / \mathrm{cm}^{-1}$ : 1775, 1794 (C=O), 2210 (C=N). ${ }^{1} \mathrm{H}$ NMR (300 MHz, DMSO/CCl, $\left.1 / 3\right) \delta 1.26$ (d, J=6.7 Hz, 6H, $\left.\mathrm{CH}_{3},{ }^{i} \mathrm{Pr}\right), 1.43-$ $1.50\left(\mathrm{~m}, 3 \mathrm{H},\left(\mathrm{CH}_{3}\right)_{2} \mathrm{CO}\right), 1.51-1.59\left(\mathrm{~m}, 3 \mathrm{H},\left(\mathrm{CH}_{3}\right)_{2} \mathrm{CO}\right), 2.02-2.42\left(\mathrm{~m}, 2 \mathrm{H}, \mathrm{CH}_{2}\right.$ in lactone, $\left.2 \mathrm{H}, \mathrm{CH}_{2} \mathrm{CH}_{2} \mathrm{CH}_{2}\right), 2.52-$ $2.72\left(\mathrm{~m}, 2 \mathrm{H}, \mathrm{CH}_{2}\right.$ in lactone), 2.78-2.94 (m, 2H, $\left.\mathrm{CH}_{2} \mathrm{CH}_{2} \underline{\mathrm{CH}}_{2}\right), 2.94-3.15\left(\mathrm{~m}, 2 \mathrm{H}, \mathrm{CH}_{2} \mathrm{CH}_{2} \mathrm{CH}_{2}, 1 \mathrm{H}, \mathrm{CH},{ }^{i} \mathrm{Pr}\right), 4.64-$ $4.90\left(\mathrm{~m}, 2 \mathrm{H}, \mathrm{CH}_{2} \mathrm{~N}\right), 4.93-5.21(\mathrm{~m}, 1 \mathrm{H}, \mathrm{HCO}), 5.54\left(\mathrm{~s}, 2 \mathrm{H}, \mathrm{CH}_{2} \mathrm{O}\right), 8.01-8.13(\mathrm{~m}, 1 \mathrm{H},=\mathrm{CH}-\mathrm{N})$. Anal. calcd. for $\mathrm{C}_{22} \mathrm{H}_{22} \mathrm{~N}_{5} \mathrm{O}_{5}$ : C 60.54; $\mathrm{H}$ 5.08; N 16.05\%. Found: C 60.71; H 4.90; N 16.22\%.

(R)-3,3,8-trimethyl-6-(\{1-[2-(4-methyl-5-oxocyclohex-3-en-1-yl)allyl]-1H-1,2,3-triazol-4-yl\}methoxy)-3,4-

dihydro-1H-pyrano[3,4-c]pyridine-5-carbonitrile (17). Yield 55\%, mp 59-60 ${ }^{\circ} \mathrm{C},[\alpha]_{\mathrm{D}}^{20}-21.44\left(\mathrm{C} 1.068 \mathrm{CH}_{2} \mathrm{Cl}_{2}\right)$. IR v/cm ${ }^{-1}$ : 1706 (C=O), 2203 (C=N). ${ }^{1} \mathrm{H}$ NMR (300 MHz, DMSO/CCl, $\left.1 / 3\right) \delta 1.27\left(\mathrm{~s}, 6 \mathrm{H}, \mathrm{OC}\left(\mathrm{CH}_{3}\right)_{2}\right), 1.63-1.78(\mathrm{~m}$, $\left.3 \mathrm{H}, \mathrm{CH}_{3}\right), 2.15-2.56\left(\mathrm{~m}, 7 \mathrm{H}, \mathrm{CH}_{2}\right.$ in carbocycle, $\left.\mathrm{N}=\mathrm{C}-\mathrm{CH}_{3}\right), 2.56-2.71\left(\mathrm{~m}, 1 \mathrm{H}, \mathrm{CH}\right.$ in carbocycle), $2.74\left(\mathrm{~s}, 2 \mathrm{H}, \mathrm{CH}_{2}\right.$ in pyran), $4.61\left(\mathrm{~s}, 2 \mathrm{H}, \mathrm{OCH}_{2}\right.$ in pyran), $4.94\left(\mathrm{~s}, 1 \mathrm{H}^{\mathrm{b}},=\mathrm{CH}_{2}\right), 5.07\left(\mathrm{~s}, 2 \mathrm{H}, \mathrm{NCH}_{2}\right), 5.08\left(\mathrm{~s}, 1 \mathrm{H}^{\mathrm{a}},=\mathrm{CH}_{2}\right), 5.51(\mathrm{~s}, 2 \mathrm{H}$, $\left.\mathrm{OCH}_{2}\right)$, 6.61-6.80 (m, 1H, CH=C-C=O), 7.98 (s, $\left.1 \mathrm{H},=\mathrm{N}-\mathrm{CH}\right) .{ }^{13} \mathrm{C} \mathrm{NMR}\left(75 \mathrm{MHz}, \mathrm{DMSO} / \mathrm{CCl}_{4}, 1 / 3\right) \delta 15.1,20.7$, 25.7, 30.4, 37.6, 37.9, 42.0, 52.5, 59.2, 59.4, 69.0, 93.0, 113.2, 113.5, 121.0, 124.2, 134.4, 142.1, 143.0, 145.5, 148.7, 155.2, 160.8, 196.5. Anal. calcd. for $\mathrm{C}_{25} \mathrm{H}_{31} \mathrm{~N}_{5} \mathrm{O}_{3}$ : C 66.79; $\mathrm{H} 6.95 ; \mathrm{N} \mathrm{15.58 \% .} \mathrm{Found:} \mathrm{C} \mathrm{66.60;} \mathrm{H} 6.84 ; \mathrm{N}$ $15.77 \%$. 


\section{Acknowledgements}

The work was supported by the Science Committee of RA, in the frames of the research project № 20TTWS$1 \mathrm{D009.}$

\section{Supplementary Material}

The copies of ${ }^{1} \mathrm{H}$ and ${ }^{13} \mathrm{C}$ NMR spectra for all new synthesized compounds have been submitted along with the manuscript.

\section{References}

1. Toguchi, Sh.; Hirose, T.; Yorita, K.; Fukui, K.; Barry, Sh. K.; Omura, S.; Sunazuka, T. Chem. Pharm. Bull. 2016, 64, 695-703. https://doi.org/10.1248/cpb.c15-00867

2. Motornov, V. A., Tabolin, A. A.; Novikov, R. A.; Nelyubina, Y. V.; loffe, S. L.; Smolyar, I. V.; Nenajdenko, V. G. Eur. J. Org. Chem. 2017, 46, 6851-6858. https://doi.org/10.1002/ejoc.201701338

3. Su, Ch.-Li; Tseng, Ch.-L.; Ramesh, Ch.; Liu, H.-Sh.; Huang, Ch.-Y. F.; Yao, Ch.-F. Eur. J. Med. Chem. 2017, 132, 90-107. https://doi.org/10.1016/i.ejmech.2017.03.034

4. Ruddarraju, R. R.; Murugulla, A. Ch.; Kotla, R.; Tirumalasetty, M. Ch.; Wudayagiri, R.; Donthabakthuni, Sh.; Majoru, R.; Baburao, K.; Parasa, L. S. Eur. J. Med. Chem. 2017, 123, 379-396. https://doi.org/10.1016/j.ejmech.2016.07.024

5. Teixeira, R. R.; Gazolla, P. A.; Silva, A. M.; Gonçalves Borsodi M. P.; Bergmann, B. R.; Ferreira, R. S.; Vaz, B. G.; Vasconcelos, G. A.; Lima, W. P. Eur. J. Med. Chem. 2018, 146, 274-286. https://doi.org/10.1016/j.ejmech.2018.01.046

6. Jiang, T.; Yang, Xia.; Yang, Xin.; Yuan, M.; Zhang, T.; Zhang, H.; Li, M. Org. Biomol. Chem. 2016, 14, 52725281.

https://doi.org/10.1039/C6OB00659K

7. Dubovis, M. V.; Rudakov, G. F.; Kulagin, A. S.; Tsarkova, K. V.; Popkov, S. V.; Goloveshkin, A. S.; Cherkaev, G. V. Tetrahedron 2018, 74, 672-683. https://doi.org/10.1016/j.tet.2017.12.043

8. Fukushima, H.; Ikegami, D.; Kuroda, C.; Kobayashi, K. Chem. Pharm. Bull. 2018, 66, 568-574. https://doi.org/10.1248/cpb.c18-00077

9. Henrion, S.; Mace, A.; Vallejos, M. M.; Roisnel, T.; Carboni, B.; Villagordo, J. M.; Carreaux, F. Org. Biomol. Chem. 2018, 16, 1672-1678. https://doi.org/10.1039/C80B00101D

10. Shingaki, M.; Wauke, T.; Ahmadi, P.; Tanaka J. Chem. Pharm. Bull. 2016, 64, 272-275. https://doi.org/10.1248/cpb.c15-00726

11. Rasyid, F. A.; Fukuyoshi, Sh.; Ando, H.; Miyake, K.; Atsumi, T.; Fujie, T.; Saito, Y.; Goto, M.; Shinya, T.; Mikage, M.; Sasaki, Y.; Nakagawa-Goto, K. Chem. Pharm. Bull. 2017, 65, 116-120. 
https://doi.org/10.1248/cpb.c16-00775

12. Piao, D.; Kim, T.; Zhang, H. Y.; Choi, H. G.; Lee, Ch. S.; Choi, H. J.; Chang, H. W.; Woo, Mi-H.; Son, J.-K. Chem. Pharm. Bull. 2016, 64, 276-281.

https://doi.org/10.1248/cpb.c15-00780

13. Zhao, S.-M.; Chou, G.-X.; Yang, Q.-Sh.; Wang, W.; Zhou, J.-L. Org. Biomol. Chem. 2016, 14, 3510-3520. https://doi.org/10.1039/C6OB00139D

14. Spivak, A. Yu.; Nedopekina, D. A.; Galimshina, Z. R.; Khalitova, R. R.; Sadretdinova, Z. R.; Gubaidullin, R. R.; Odinokov, V. N. Arkivoc 2018, vii, 1-19. https://doi.org/10.24820/ark.5550190.p010.632

15. Quan, Z.; Xu, Q.; Zhang, Z.; Da, Y.; Wang, X. Tetrahedron 2013, 69, 881-887. https://doi.org/10.1016/j.tet.2012.10.097

16. Demko, Z. P.; Sharpless, K. B. Angew. Chem. Int. Ed. 2002, 41, 2113-2116. https://doi.org/10.1002/1521-3773(20020617)41:12<2113::AID-ANIE2113>3.0.CO;2-Q

17. Obniska, J.; Góra, M.; Rapacz, A.; Sałat, K.; Rybka, S.; Abram, M.; Jakubiec, M.; Kamiński, K. Arch. Pharm. 2020, 354, 2000225.

https://doi.org/10.1002/ardp.202000225

18. Upadhayaya, R. S.; Sinha, N.; Jain, S.; Kishore, N.; Chandra, R.; Arora S. K., Bioorg. Med. Chem. 2004, 12, 2225-2238.

https://doi.org/10.1016/j.bmc.2004.02.014

19. Selvakumar, B.; Vaidyanathan, S. P.; Subbiah, M.; Elango, K. P. Arkivoc 2017, iv, $353-364$. https://doi.org/10.24820/ark.5550190.p010.037

20. Filipova, A.; Marek, J.; Havelek, R.; Pejchal, J.; Jelicova, M.; Cizkova, J.; Majorosova, M.; Muckova, L.; Kucera, T.; Prchal, L.; Psotka, M.; Zivna, N.; Koutova, D.; Sinkorova, Z.; Rezacova, M.; Tichy, A. Molecules 2020, 25, 532-532.

https://doi.org/10.3390/molecules25030532

21. Patel, R.V.; Park, S.W. Mini Rev. Med. Chem. 2013, 13, 1579-1601.

https://doi.org/10.2174/13895575113139990073

22. Choudhary, P.; Kumar, R.; Verma, K.; Singh D.; Yadav, V.; Chhillar, A.K.; Sharma, G.L.; Chandra, R. Bioorg. Med. Chem. 2006, 14, 1819-1826.

https://doi.org/10.1016/j.bmc.2005.10.032

23. Sirakanyan, S. N.; Kartsev, V. G.; Spinelli, D.; Geronikaki, A.; Petrou, A.; Ivanov, M.; Glamoclija, J.; Sokovic, M.; Hakobyan, E.; Hovakimyan, A. A. Arch. Pharm. 2021, 354, 2000208.

https://doi.org/10.1002/ardp.202000208

24. Sirakanyan, S. N.; Akopyan, E. K.; Paronikyan, R. G.; Nazaryan, I. M.; Akopyan, A. G.; Ovakimyan, A. A. Pharm. Chem. J. 2019, 53, 495-499.

https://doi.org/10.1007/s11094-019-02026-8

25. Sirakanyan, S. N.; Noravyan, A. S.; Dzhagatspanyan, I. A.; Nazaryan, I. M.; Ovakimyan, A. A.; Akopyan, A. G.; Avetisyan, N. G. Pharm. Chem. J. 2013, 46, 591-594.

https://doi.org/10.1007/s11094-013-0852-2

26. Paronikyan, E. G.; Sirakanyan, S. N.; Hovhannisyan, A. X.; Noravyan, A. S.; Samvelyan, V. M.; Janpolandyan, E. G.; Shirinyan, E. A. R.A. Patent 619 A2, 1995; Bulletin 16.03.1999.

27. Ghochikyan, T. V.; Muzalevskiy, V. M.; Samvelyan, M. A.; Galstyan, A. S.; Nenajdenko, V. G. Mendeleev Commun. 2016, 26, 11-13.

https://doi.org/10.1016/j.mencom.2016.01.005 
28. Baldwin, J. E. J. Chem. Soc., Chem. Commun. 1976, 734-736.

https://doi.org/10.1039/C39760000734

29. Alabugin, I. V. ; Gilmore, K. Chem. Commun. 2013, 49, 11246-11250.

https://doi.org/10.1039/C3CC43872D

30. Houk, K. N.; Jabbari, A.; Hall, H. K.; Aleman C. J. Org. Chem. 2008, 73, 2674-2678. https://doi.org/10.1021/jo702567v

31. Baroudi, A.; Mauldin, J.; Alabugin, I. V. J. Am. Chem. Soc. 2010, 132, 967-979. https://doi.org/10.1021/ja905100u

32. Galstyan, A. S.; Martiryan, A. I.; Grigoryan, K. R.; Ghazaryan, A. G.; Samvelyan, M. A.; Ghochikyan, T. V.; Nenajdenko, V. G. Molecules 2018, 23, 2991-3002.

https://doi.org/10.3390/molecules23112991

33. Rosowsky, A.; Papathansopoulos, N. J. Med. Chem. 1974, 17, 1272-1276.

https://doi.org/10.1021/jm00258a008

34. Paronikyan, E. G.; Sirakanyan, S. N.; Lindeman, S. V.; Aleksanyan, M.S.; Karapetyan, A. A.; Noravyan, A. S.; Struchkov, Yu. T. Chem. Heterocycl. Compd. 1989, 25, 953-958.

https://doi.org/10.1007/BF00479620

35. Galstyan, A. S.; Ghochikyan, T. V.; Samvelyan, M. A.; Frangyan, V. R.; Tamazyan, R. A.; Ayvazyan, A. G. Chem. Select 2019, 4, 12386-12390.

https://doi.org/10.1002/slct.201802243 